Research Article

\title{
Some Fractional Operators with the Generalized Bessel-Maitland Function
}

\author{
R. S. Ali, ${ }^{1}$ S. Mubeen $\left(\mathbb{D},{ }^{1}\right.$ I. Nayab, ${ }^{1}$ Serkan Araci $\left(\mathbb{D},{ }^{2}\right.$ G. Rahman ${ }^{(D)},{ }^{3}$ and K. S. Nisar ${ }^{4}$ \\ ${ }^{1}$ Department of Mathematics, University of Sargodha, Sargodha, Pakistan \\ ${ }^{2}$ Department of Economics, Faculty of Economics, Administrative and Social Sciences, Hasan Kalyoncu University, \\ TR-27410 Gaziantep, Turkey \\ ${ }^{3}$ Department of Mathematics, Shaheed Benazir Bhutto University, Sheringal, Pakistan \\ ${ }^{4}$ Department of Mathematics, College of Arts and Sciences, Prince Sattam Bin Abdulaziz University, \\ Wadi Aldawaser 11991, Saudi Arabia
}

Correspondence should be addressed to Serkan Araci; serkan.araci@hku.edu.tr

Received 21 April 2020; Revised 13 June 2020; Accepted 29 June 2020; Published 24 July 2020

Guest Editor: Qasem M. Al-Mdallal

Copyright $\odot 2020$ R. S. Ali et al. This is an open access article distributed under the Creative Commons Attribution License, which permits unrestricted use, distribution, and reproduction in any medium, provided the original work is properly cited.

In this paper, we aim to determine some results of the generalized Bessel-Maitland function in the field of fractional calculus. Here, some relations of the generalized Bessel-Maitland functions and the Mittag-Leffler functions are considered. We develop Saigo and Riemann-Liouville fractional integral operators by using the generalized Bessel-Maitland function, and results can be seen in the form of Fox-Wright functions. We establish a new operator $\mathscr{Z}_{\nu, \eta, \eta, c, c, w, a^{+}}^{\mu, \xi} \phi$ and its inverse operator $D_{\nu, \eta, \eta, \rho, \gamma, w, a^{+}}^{\mu, \xi} \phi$, involving the generalized Bessel-Maitland function as its kernel, and also discuss its convergence and boundedness. Moreover, the Riemann-Liouville operator and the integral transform (Laplace) of the new operator have been developed.

\section{Introduction}

During the last few years, many types of research studies developed the class of generalized fractional integrals containing a variety of special functions [1-5]. Watson [6] discussed applications of the Bessel function with some fields of applied sciences, biology, chemistry, physical sciences, and engineering. The generalization and extensions of the Bessel-Maitland function [7-14] dealt with special cases that gave useful results in different areas of mathematics. The recent work in the field of fractional calculus theory, differential equations of the Mittag-Leffler function, Sturm-Liouville problems in theoretical sense, Gronwall's inequality, and exponential kernels of the differential operator [15-18] have found many applications in various subfields of mathematical analysis.

The series representation of the Bessel-Maitland function [19] is defined as

$$
J_{\beta}^{\alpha}(s)=\sum_{n=0}^{\infty} \frac{(-s)^{n}}{n ! \Gamma(\alpha n+\beta+1)}=\phi(\alpha, \beta+1 ;-s)
$$

The generalization of the Bessel-Maitland function introduced by Singh et al. [7] is

$$
J_{\beta, q}^{\alpha, \gamma}(s)=\sum_{n=0}^{\infty} \frac{(\gamma)_{q n}(-s)^{n}}{\Gamma(\alpha n+\beta+1) n !}
$$

where $\quad \alpha, \gamma, \beta \in \mathbb{C}, \quad \mathfrak{R}(\alpha) \geq 0, \mathfrak{R}(\beta) \geq-1, \mathfrak{R}(\gamma) \geq 0, \quad$ and $q \in(0,1) \cup \mathbb{N}$.

The extended Bessel-Maitland function investigated in [20] is

$$
J_{\beta, \gamma, \delta}^{\alpha, q, p}(s)=\sum_{n=0}^{\infty} \frac{(\gamma)_{q n}(-s)^{n}}{\Gamma(\alpha n+\beta+1)(\delta)_{p n}},
$$

where $\quad \alpha, \gamma, \beta, \delta \in \mathbb{C}, \quad \mathfrak{R}(\alpha)>0, \mathfrak{R}(\beta)>-1, \quad \mathfrak{R}(\gamma)>0$, $\mathfrak{R}(\delta) \geq 0, p, q>0$, and $q<\Re(\alpha)+p$. 
The Saigo fractional integral operators are defined [21] for $s>0, a, c, d \in \mathbb{C}$, and $\mathfrak{R}(a)>0$ :

$$
\begin{aligned}
\left(\mathscr{F}_{0 *+}^{a, c, d} g\right)(s)= & \frac{s^{-a-c}}{\Gamma(a)} \int_{0}^{s}(s-\tau)^{a-1} \\
& \cdot{ }_{2} R_{1}\left(a+c,-d ; a ;\left(1-\frac{\tau}{s}\right)\right) g(\tau) \mathrm{d} \tau \\
\left(\mathscr{F}_{0-}^{a, c, d} g\right)(s)= & \frac{1}{\Gamma(a)} \int_{s}^{\infty}(\tau-s)^{a-1} \tau^{-a-c} \\
& \cdot{ }_{2} R_{1}\left(a+c,-d ; a ;\left(1-\frac{s}{\tau}\right)\right) g(\tau) \mathrm{d} \tau .
\end{aligned}
$$

Samko et al. [22] defined the Riemann-Liouville fractional operators for $\mathfrak{R}(a)>0$ and $n=[\operatorname{Re}(a)]+1$ as

$$
\begin{aligned}
& \left(\mathscr{F}_{0+}^{a} g\right)(s)=\frac{1}{\Gamma(a)} \int_{0}^{s}(s-\tau)^{a-1} g(\tau) \mathrm{d} \tau, \\
& \left(\mathscr{D}_{0+}^{a} \phi\right)(s)=\left(\frac{\mathrm{d}}{\mathrm{d} s}\right)^{n}\left(\mathscr{F}_{0+}^{n-a} \phi\right)(s) .
\end{aligned}
$$

The Gauss hypergeometric function defined by Saigo [21] for all $a, c, d \in \mathbb{C}, a \neq 0$, and $|s|<1$ is

$$
{ }_{2} R_{1}(b,-d ; a ; s)=\sum_{n=0}^{\infty} \frac{(b)_{n}(-d)_{n} s^{n}}{(a)_{n} n !},
$$

where $(b)_{n},(-d)_{n}$, and $(a)_{n}$ are Pochhammer's symbols.

Pochhammer's symbols defined by Petojevic [23] are

$$
(s)_{n}= \begin{cases}s(s+1)(s+2) \cdots(s+n-1), & \text { for } n \geq 1, \\ 1, & \text { for } n=0, s \neq 0,\end{cases}
$$

where $s \in \mathbb{C}$ and $n \in \mathbb{N}$, and in gamma form, they can be written as

$$
(s)_{n}=\frac{\Gamma(s+n)}{\Gamma(s)} .
$$

The beta function is defined as given in [23], for $\mathfrak{R}(y)>0$ and $\mathfrak{R}(z)>0$, and also expressed in the gamma form, respectively:

$$
\begin{aligned}
& \beta(y, z)=\int_{0}^{1} g^{y-1}(1-g)^{z-1} \mathrm{~d} g \\
& \beta(y, z)=\frac{\Gamma(y) \Gamma(z)}{\Gamma(y+z)}
\end{aligned}
$$

The gamma function is defined [23] for $\mathfrak{R}(u)>0$ as

$$
\Gamma(u)=\int_{0}^{\infty} g^{u-1} e^{-g} \mathrm{~d} g .
$$

The generalized hypergeometric function is defined by Rainville [24]:

$$
{ }_{k} R_{r}\left(c_{1}, \ldots, c_{k}, q_{1}, \ldots, q_{r} ; s\right)=\sum_{n=0}^{\infty} \frac{\left(c_{1}\right)_{n}, \ldots,\left(c_{k}\right)_{n}}{\left(q_{1}\right)_{n}, \ldots,\left(q_{r}\right)_{n}} \frac{s^{n}}{n !},
$$

where $\quad c_{i}, q_{j} \in \mathbb{C}, \quad q_{j} \neq 0,-1, \ldots,(i=-1,-2, \ldots, k ; j=$ $-1,-2, \ldots, r)$.

The generalized Fox-Wright function is defined as [25]

$$
{ }_{r} \psi_{s}(g)={ }_{r} \psi_{s}\left[\begin{array}{l}
\left(y_{j}, p_{j}\right)_{1, r} \\
\left(z_{i}, q_{i}\right)_{1, s}
\end{array} \mid g\right] \equiv \sum_{n=0}^{\infty} \frac{\prod_{j=1}^{r} \Gamma\left(y_{j}+p_{j} n\right)}{\prod_{i=1}^{s} \Gamma\left(z_{i}+q_{i} n\right)} \frac{g^{n}}{n !},
$$

where $g \in \mathbb{C}, \quad y_{j}, z_{i} \in \mathbb{C}, \quad$ and $\quad p_{j}, q_{i} \in \mathbb{R}(j=1,2, \ldots$, $r ; i=1,2, \ldots, s)$.

The Gauss hypergeometric function in the gamma form can be written as

$$
{ }_{2} R_{1}(a, s ; d ; 1)=\frac{\Gamma(d) \Gamma(d-a-s)}{\Gamma(d-a) \Gamma(d-s)}, \quad \mathfrak{R}(d-a-s)>0 .
$$

The Laplace transform of function $f(z)$ is defined as

$$
\mathscr{L}[f(t)]=f(s)=\int_{0}^{\infty} e^{-s t} f(t) \mathrm{d} t
$$

Dirichlet formula (Fubini's theorem) [22] is given by

$$
\int_{d}^{c} \mathrm{~d} y \int_{y}^{c} g(y, \tau) \mathrm{d} \tau=\int_{d}^{c} \mathrm{~d} \tau \int_{d}^{\tau} g(y, \tau) \mathrm{d} y .
$$

Definition 1. The generalization of the generalized Bessel-Maitland function is defined and investigated as

$$
\mathscr{J}_{\nu, \eta, \rho, \gamma}^{\mu, \xi, m, \sigma}(s)=\sum_{n=0}^{\infty} \frac{(\eta)_{\xi n}(\gamma)_{\sigma n}(-s)^{n}}{\Gamma(\mu n+\nu+1)(\rho)_{m n}},
$$

where $\mu, \nu, \eta, \rho, \gamma \in \mathbb{C}, \quad \mathfrak{R}(\mu)>0, \quad \mathfrak{R}(\nu) \geq-1, \quad \mathfrak{R}(\eta)>0$, $\mathfrak{R}(\rho)>0, \mathfrak{R}(\gamma)>0, \xi, m, \sigma \geq 0$, and $m, \xi>\mathfrak{R}(\mu)+\sigma$.

The following notation is used in our results:

$$
{ }_{\mu, \nu}^{\gamma, \sigma} Q_{\rho, m ; n}^{\eta, \xi}=\sum_{n=0}^{\infty} \frac{(\eta)_{\xi n}(\gamma)_{\sigma n}(-1)^{n}}{\Gamma(\mu n+\nu+1)(\rho)_{m n}} .
$$

Definition 2. The extension of generalized Bessel-Maitland function (19) in multivariable function can be defined for $\mu_{j}, \nu, \eta_{j}, \rho_{j}, \gamma_{j} \in \mathbb{C}, \quad \mathfrak{R}\left(\mu_{j}\right)>0, \quad \mathfrak{R}(\nu)>-1, \quad \mathfrak{R}\left(\eta_{j}\right)>0$, $\mathfrak{R}\left(\rho_{j}\right)>0, \mathfrak{R}\left(\gamma_{j}\right)>0, \xi_{j}, m_{j}, \sigma_{j} \geq 0$, and $m_{j}, \xi_{j}>\mathfrak{R}\left(\mu_{j}\right)+\sigma_{j}$, $j=1, \ldots, r$, as

$$
\begin{aligned}
J_{v ; \eta_{j} ; \rho_{j} ; \gamma_{j}}^{\mu_{j} ; \xi_{j} ; m_{j} ; \sigma_{j}}\left(s_{1} \ldots s_{r}\right) & =J_{v ; \eta_{1}, \ldots, \eta_{r} ; \rho_{j} ; \gamma_{1}, \ldots, \gamma_{r}}^{\mu_{1}, \ldots, \mu_{r} ; \xi_{1}, \ldots, \xi_{r} ; m_{1}, \ldots, m_{r} ; \sigma_{1}, \ldots, \sigma_{r}}\left(s_{1} \ldots s_{r}\right) \\
& =\sum_{n_{1}, \ldots, n_{r}=0}^{\infty} \frac{\left(\eta_{1}\right)_{\xi_{1} n_{1}}, \ldots,\left(\eta_{r}\right)_{\xi_{r} n_{r}}\left(\gamma_{1}\right)_{\sigma_{1} n_{1}}, \ldots,\left(\gamma_{r}\right)_{\sigma_{r} n_{r}}}{\Gamma\left(\nu+1+\sum_{j=1}^{r} n_{j} \mu_{j}\right)\left(\rho_{1}\right)_{m_{1} n_{1}}, \ldots,\left(\rho_{r}\right)_{m_{r} n_{r}}}\left(-s_{1}\right)^{n_{1}} \ldots\left(-s_{r}\right)^{n_{r}} .
\end{aligned}
$$


Remark 1 . On setting $j=1$ in equation (21), we get generalized Bessel-Maitland function (19).

Definition 3. An integral operator which involves generalized Bessel-Maitland function (19) as its kernel is defined for $\mu, \nu, \eta, w, \gamma, \rho \in \mathbb{C}, \quad \mathfrak{R}(\mu)>0, \quad \mathfrak{R}(\nu) \geq-1, \quad \mathfrak{R}(\eta)>0$, $\mathfrak{R}(w)>0, \mathfrak{R}(\gamma)>0, \mathfrak{R}(\rho)>0, \xi, m, \sigma \geq 0$, and $m, \xi>\mathfrak{R}(\mu)+$ $\sigma$ as follows:

$$
\left(\mathscr{E}_{\nu, \eta, \rho, \gamma, w, a^{+}}^{\mu, \xi, m, \sigma} \phi\right)(s)=\int_{a}^{s}(s-\tau)^{\nu} \mathscr{J}_{\nu, \eta, \rho, \gamma}^{\mu, \xi, m, \sigma}\left[w(s-\tau)^{\mu}\right] \phi(\tau) \mathrm{d} \tau .
$$

Remark 2. If we put $w=0$ and replace $v$ by $v-1$, then it will become a left-sided Riemann-Liouville fractional integral operator.

The new fractional operator (22) can be discussed to improve the results of some inequalities such as Polya-Szego inequality, Chebyshev inequality, and Hadamard inequality in the field of analysis.

Definition 4. The left inverse operator of integral operator (22), for $\mu, \nu, \eta, w, \gamma, \rho \in \mathbb{C}, \mathfrak{R}(\mu)>0, \mathfrak{R}(\nu) \geq-1, \mathfrak{R}(\eta)>0$, $\mathfrak{R}(w)>0, \mathfrak{R}(\gamma)>0, \mathfrak{R}(\rho)>0, \xi, m, \sigma \geq 0, m, \xi>\mathfrak{R}(\mu)+\sigma$, and $n=[\nu]$ as $n-\nu>0$ is defined as follows:

$$
\begin{aligned}
\left(D_{\nu, \eta, \rho, \gamma, w, a^{+}}^{\mu, \xi, m, \sigma} \phi\right)(s)= & \left(\frac{\mathrm{d}^{n}}{\mathrm{~d} s^{n}}\left(\mathscr{Z}_{\nu-n, \eta, \rho, \gamma, w, a^{+}}^{\mu, \xi, m, \sigma} \phi\right)\right)(s) \\
= & \frac{\mathrm{d}^{n}}{\mathrm{~d} s^{n}} \int_{a}^{s}(s-\tau)^{n-\nu} \mathscr{J}_{n-\nu, \eta, \rho, \gamma}^{\mu, \xi, m, \sigma}\left[w(s-\tau)^{\mu}\right] \\
& \cdot \phi(\tau) \mathrm{d} \tau .
\end{aligned}
$$

Remark 3. If we put $w=0$ and replace $v$ by $v-1$, then equation (23) becomes the Riemann-Liouville fractional differential operator.

Remark 4. If we replace $\sigma=0, \eta=-\eta, \xi=\rho=m=1$, and $v=v-1$ in equation (23), we get

$$
\left(D_{\nu-1,-\eta, 1, \gamma, w, a^{+}}^{\mu, 1,1,0} \phi\right)(s)=\left(\mathbf{D}_{\mu, \nu, w, a^{+}}^{\eta} \phi\right)(s)
$$

where the inverse operator $\left(\mathbf{D}_{\mu, \nu, w, a^{+}}^{\eta} \phi\right)(s)$ is described and discussed by Polito and Tomovski in [26].

\section{Relation with the Bessel-Maitland and the Mittag-Leffler Functions}

In this section, we discuss some special cases of the generalized Bessel-Maitland function and developed its relations with generalized Mittag-Leffler functions:

(i) On replacing $\sigma=0$ in equation (19), we obtain the relation

$$
\mathcal{J}_{\nu, \eta, \rho, \gamma}^{\mu, \xi, m, 0}(s)=J_{\nu, \eta, \rho}^{\mu, \xi, m}(s)
$$

where $J_{v, \eta, \rho}^{\mu, \xi, m}(z)$ is the generalized Bessel-Maitland function investigated in [20].

(ii) On replacing $\sigma=0$ and $m=\rho=1$ in equation (19), we obtain the relation

$$
\mathcal{J}_{v, \eta, 1,1}^{\mu, \xi, 1,0}(s)=J_{v, \eta}^{\mu, \xi}(s)
$$

where $J_{v, \eta}^{\mu, \xi}(s)$ is the generalization of Bessel-Maitland function defined by Singh et al. [7].

(iii) On replacing $\sigma=\xi=0$ and $m=\rho=1$ in equation (19), we obtain the relation [19]

$$
\mathscr{J}_{\nu, \eta, 1, \gamma}^{\mu, 0,1,0}(s)=J_{\nu}^{\mu}(s)
$$

(iv) On setting $\sigma=0$ and replacing $v$ by $v-1$ in equations (19) and (22), then we have

$$
\begin{gathered}
\mathscr{J}_{\nu-1, \eta, \rho, \gamma}^{\mu, \xi, m, 0}(-s)=\mathscr{E}_{\mu, \nu, m}^{\eta, \rho, \xi}(s), \\
\left(\mathscr{Z}_{\nu-1, \eta, \rho, \gamma, w, a^{+}}^{\mu, \xi, m, 0}\right)(s)=\left(\mathscr{F}_{\mu, \nu, m, w, a^{+}}^{\eta, \rho, \xi} \phi\right)(w),
\end{gathered}
$$

where $\mathscr{E}_{\mu, \nu, m}^{\eta, \rho, \xi}(s)$ is the Mittag-Leffler function investigated by Salim and Faraj [27].

(v) On setting $\sigma=0$ and $\rho=m=1$ and replacing $\nu$ by $\nu-1$ in equation (19), then we have

$$
\begin{gathered}
\mathscr{J}_{\nu-1, \eta, 1, \gamma}^{\mu, \xi, 1,0}(-s)=\mathscr{E}_{\mu, \nu}^{\eta, \xi}(s), \\
\left(\mathscr{Z}_{\nu-1, \eta, 1, \gamma, w, a^{+}}^{\mu, \xi, 1,0}\right)(s)=\mathscr{E}_{a^{+} ; \mu, \nu}^{w ; \eta, \xi}(s),
\end{gathered}
$$

where $\mathscr{E}_{\mu, \nu}^{\eta, \rho}(s)$ is the Mittag-Leffler function defined by Shukla and Prajapati [28] and $\mathscr{E}_{a^{+} ; \mu, \nu}(s)$ is described by Srivastava and Tomovski in [29].

(vi) On setting $\sigma=0$ and $\xi=m=\rho=1$ and replacing $\nu$ by $\nu-1$ in equations (19) and (22), then we have

$$
\begin{aligned}
\mathscr{F}_{\nu-1, \eta, 1, \gamma}^{\mu, 1,1,0}(-s) & =\mathscr{E}_{\mu, \nu}^{\eta}(s), \\
\left(\mathscr{Z}_{\nu-1, \eta, 1, \gamma, w, a^{+}}^{\mu, 1,1,0} \phi\right)(s) & =\mathscr{F}^{*}(\mu, \nu ; \eta ; w) \phi(s),
\end{aligned}
$$

where $\mathscr{E}_{\mu, \nu}^{\eta}(s)$ is defined by Prabhakar in [30] and described the integral

$$
\mathscr{F}^{*}(\mu, v ; \eta ; w) \phi(s) .
$$

(vii) On setting $\sigma=0$ and $\xi=m=\eta=\rho=\gamma=1$ and replacing $\nu$ by $\nu-1$ in equation (19), then we have

$$
\mathscr{J}_{\nu-1,1,1, \gamma}^{\mu, 1,1,0}(-s)=\mathscr{E}_{\mu, \nu}(s),
$$

where $\mathscr{E}_{\mu, \nu}(s)$ is the Mittag-Leffler function discussed by Wiman [31].

(viii) On setting $\sigma=0, \xi=m=\eta=\rho=\gamma=1$, and $\nu=0$ in equation (19), then we have

$$
\mathscr{J}_{0,1,1, \gamma}^{\mu, 1,1,0}(-s)=\mathscr{E}_{\mu}(s),
$$


where $\mathscr{E}_{\mu}(s)$ is the Mittag-Leffler function introduced in [32].

\section{Convergence and Boundedness of the New Fractional Integral Operator}

In this section, we discuss the convergence and boundedness of the fractional integral operator involving the generalized Bessel-Maitland function as its kernel in the form of a theorem.

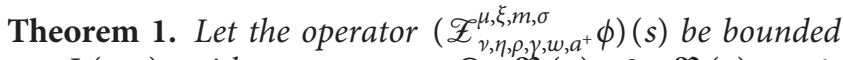
on $L(a, c)$ with $\mu, \nu, \eta, w, \gamma \in \mathbb{C}, \Re(\mu)>0, \quad \Re(\nu) \geq-1$, $\Re(\eta)>0, \quad \mathfrak{R}(w)>0, \quad \mathfrak{R}(\gamma)>0, \quad \xi, m, \sigma \geq 0, \quad$ and $m, \xi>\Re(\mu)+\sigma$; then, the following relation holds:

$$
\left\|\left(\mathscr{Z}_{\gamma, \eta, \rho, \gamma, w, a^{+}}^{\mu, \xi, m, \sigma}\right)\right\|_{\mathcal{c}} \leq B\|\phi\|_{\mathcal{C}},
$$

where

$$
\begin{aligned}
B= & (c-a)^{\operatorname{Re}(\nu)} \sum_{n=0}^{\infty} \frac{\left|(\eta)_{\xi n}\right|\left|(\gamma)_{\sigma n}\right|}{\left|(\rho)_{m n}\right|} \\
& \cdot \frac{\left|\left(-w(c-a)^{\operatorname{Re}(\mu)}\right)^{n}\right|}{|\Gamma(\mu n+\nu+1)||\operatorname{Re}(\mu) n+\operatorname{Re}(\nu)+1|} .
\end{aligned}
$$

Proof. Let $K_{n}$ denote the $n$th term of (36); then,

$$
\begin{aligned}
\left|\frac{K_{n+1}}{K_{n}}\right|= & \left|\frac{(\eta)_{\xi n+\xi}}{(\eta)_{\xi n}}\right|\left|\frac{(\gamma)_{\sigma n+\sigma}}{(\gamma)_{\sigma n}}\right|\left|\frac{(\rho)_{m n}}{(\rho)_{m n+m}}\right|\left|\frac{\Gamma(\mu n+\nu+1)}{\Gamma(\mu n+\mu+\nu+1)}\right| \\
& \times\left|\frac{\operatorname{Re}(\mu) n+\operatorname{Re}(\nu)+1}{\operatorname{Re}(\mu)(n+1)+\operatorname{Re}(\nu)+1}\right|\left|\frac{(-1)^{n+1}}{(-1)^{n}}\right|\left|w(c-a)^{\operatorname{Re}(\mu)}\right| \\
& \approx \frac{(\xi n)^{\xi}(\sigma n)^{\sigma}\left|w(c-a)^{\operatorname{Re}(\mu)}\right|}{(\rho n)^{\rho}|n+1|\left|(|\mu| n)^{\operatorname{Re}(\mu)}\right|}, \quad \text { as } n \longrightarrow \infty .
\end{aligned}
$$

Hence, $\quad\left|K_{n+1} / K_{n}\right| \longrightarrow 0$ as $n \longrightarrow \infty$, and $\xi, \sigma<$ $\rho+\operatorname{Re}(\mu)$ which means that the right-hand side of (36) is convergent and finite under the given condition. The condition of boundedness of the integral operator $\left(\mathscr{X}_{\nu, \eta, \rho, \gamma, w, a^{+}}^{\mu, \xi, m}\right)(x)$ is discussed in the space of Lebesgue measure $L(a, c)$ of a continuous function on $(a, c)$, where $c>a$ :

$$
L(a, c)=\left\{g(x):\|g\|_{c}=\int_{a}^{c}|g(x)| \mathrm{d} x<\infty\right\} .
$$

According to equations (19) and (22), we have

$$
\begin{aligned}
\left\|\mathscr{E}_{\nu, \eta, p, \gamma, w, a^{+}}^{\mu, \xi, m, \sigma}\right\|_{c} & =\int_{a}^{c}\left|\int_{a}^{s}(s-t)^{\nu}\right| \mathcal{F}_{\nu, \eta, \eta, \gamma}^{\mu, \xi, m, \sigma}\left[w(s-t)^{\mu}\right]|\phi(t) \mathrm{d} t| \mathrm{d} s \\
& \leq \int_{a}^{c}\left[\int_{t}^{c}(s-t)^{\nu}\left|\mathcal{F}_{\nu, \eta, p, \gamma}^{\mu, \xi, m, \sigma}\left[w(s-t)^{\mu}\right]\right| \mathrm{d} s\right]|\phi(t)| \mathrm{d} t .
\end{aligned}
$$

By putting the values $s-t=y \Rightarrow \mathrm{d} s=\mathrm{d} y$, $s=c \Rightarrow y=c-t$, and $s=t \Rightarrow y=0$ in equation (39), we have

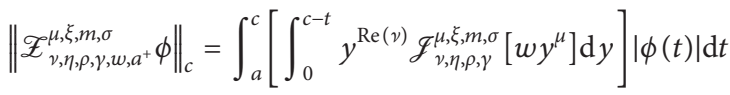

$$
\begin{aligned}
& \leq \int_{a}^{c}\left[\int_{0}^{c-a} y^{\operatorname{Re}(\nu)}\left|\mathcal{F}_{\nu, \xi, \eta, \rho, \gamma}^{\mu, \xi}\left(w y^{\mu}\right)\right| \mathrm{d} y\right]|\phi(t)| \mathrm{d} t, \\
& B=\int_{0}^{c-a} y^{\operatorname{Re}(\nu)}\left|\mathcal{F}_{\nu, \eta, \rho, \gamma}^{\mu, \xi, m, \sigma}\left(w y^{\mu}\right)\right| \mathrm{d} y, \\
& B=\sum_{n=0}^{\infty} \frac{\left|(\eta)_{\xi n}\right|\left|(\gamma)_{\sigma n}\right|\left|(-w)^{n}\right|}{\left|(\rho)_{m n}\right||\Gamma(\mu n+\nu+1)|} \int_{0}^{c-a} y^{\operatorname{Re}(\mu) n+\operatorname{Re}(\nu)} \mathrm{d} y \\
& =\sum_{n=0}^{\infty} \frac{(c-a)^{\operatorname{Re}(\nu)}\left|(\eta)_{\xi_{n}}\right|\left|(\gamma)_{\sigma n}\right|\left|\left(-w(c-a)^{\operatorname{Re}(\mu)}\right)^{n}\right|}{\left|(\rho)_{m n}\right||\Gamma(\mu n+\nu+1)||\operatorname{Re}(\mu) n+\operatorname{Re}(\nu)+1|} .
\end{aligned}
$$

Therefore,

$$
\begin{aligned}
\left\|\mathscr{Z}_{\nu, \eta, p, \gamma, \gamma, a^{+}}^{\mu, \xi, m, \sigma}\right\|_{c} & \leq \int_{a}^{c} B|\phi(t)| \mathrm{d} t \leq B\|\phi\|_{\mathcal{c}} \\
& \Rightarrow\left\|\mathscr{Z}^{\mu, \xi, \eta,,, \rho, \gamma, w, a^{+}} \phi\right\|_{c} \leq B\|\phi\|_{c^{\prime}} .
\end{aligned}
$$

\section{The Generalized Bessel-Maitland Function with Some Fractional Integral Operators}

In this section, we derive some results of Saigo fractional integral operators with the generalized Bessel-Maitland function, and these results are established in terms of the Fox-Wright function. Also, we develop the composition of Riemann-Liouville operators with the generalized Bessel-Maitland function.

Theorem 2. Let $a, c, d, \mu, v, \eta, \rho, \gamma \in \mathbb{C}$ with $\mathfrak{R}(a)>0$, $\rho>\max [0, \mathfrak{R}(c-d)], \quad \mathfrak{R}(\mu)>0, \quad \mathfrak{R}(\nu) \geq-1, \quad \mathfrak{R}(\eta)>0$, $\mathfrak{R}(\rho)>0, \mathfrak{R}(\gamma)>0, \xi, m, \sigma \geq 0$, and $m, \xi>\mathfrak{R}(\mu)+\sigma$; then, the following relation holds:

$$
\begin{aligned}
& \mathscr{F}_{0+}^{a, c, d}\left[\tau^{\rho} \mathscr{F}_{v, \eta, \rho, \gamma}^{\mu, \xi, m, \sigma}\left(\tau^{\delta}\right)\right](s)=\frac{s^{\rho-c} \Gamma(\rho)}{\Gamma(\eta) \Gamma(\gamma)} \\
& \times_{5} \psi_{4}\left[\begin{array}{l|l}
(\eta, \xi)(\gamma, \sigma)(\rho+1, \delta)(\rho+d-c+1, \delta)(1,1) & -s^{\delta} \\
(\nu+1, \mu)(\rho, m)(\rho-c+1, \delta)(a+\rho+d+1, \delta) &
\end{array}\right] .
\end{aligned}
$$


Proof. Consider the left-sided Saigo fractional integral operator (4), in which using the power function with generalized Bessel-Maitland function (19), we get

$$
\begin{aligned}
\mathscr{F}_{0+}^{a, c, d} & {\left[\tau^{\rho} \mathcal{F}_{\nu, \eta, \rho, \gamma}^{\mu, \xi, m, \sigma}\left(\tau^{\delta}\right)\right](s) } \\
= & \frac{s^{-a-c}}{\Gamma(a)} \int_{0}^{s}(s-\tau)^{a-1}{ }_{2} R_{1}\left(a+c,-d ; a ;\left(1-\frac{\tau}{s}\right)\right) \\
& \cdot\left[\tau^{\rho} \sum_{n=0}^{\infty} \frac{(\eta)_{\xi n}(\gamma)_{\sigma n}\left(-\tau^{\delta}\right)^{n}}{\Gamma(\mu n+v+1)(\rho)_{m n}}\right] \mathrm{d} \tau .
\end{aligned}
$$

By using equation (8) in equation (43), we have

$$
\begin{aligned}
& \left(\mathscr{F}_{0+}^{a, c, d}\left[\tau^{\rho} \mathscr{J}_{\nu, \eta, \rho, \gamma}^{\mu, \xi, m, \sigma}\left(\tau^{\delta}\right)\right]\right)(s) \\
& =\frac{s^{-c-1}\left[\begin{array}{l}
\gamma, \sigma \\
\mu, \nu
\end{array} Q_{\rho, m ; n}^{\eta, \xi}\right]}{\Gamma(a)} \sum_{p=0}^{\infty} \frac{(a+c)_{p}(-d)_{p}}{(a)_{p} p !} \\
& \cdot \int_{0}^{s}\left(1-\frac{\tau}{s}\right)^{a+p-1}(\tau)^{\rho+\delta n} \mathrm{~d} \tau .
\end{aligned}
$$

By putting the values $(\tau / s)=u \Rightarrow \mathrm{d} \tau=s \mathrm{~d} u, \tau=s \Rightarrow u=$ 1 , and $\tau=0 \Rightarrow u=0$ in equation (45), we obtain

$$
\begin{aligned}
\left(\mathscr{F}_{0+}^{a, c, d}\left[\tau^{\rho} \mathcal{J}_{\nu, \eta, \rho, \gamma}^{\mu, \xi, m, \sigma}\left(\tau^{\delta}\right)\right]\right)(s) & \\
= & s^{\rho-c}\left[{ }_{\mu, \nu}^{\gamma, \sigma} Q_{\rho, m ; n}^{\eta, \xi}\left(s^{\delta}\right)^{n}\right] \sum_{p=0}^{\infty} \frac{(a+c)_{p}(-d)_{p}}{\Gamma(a)(a)_{p} p !} \\
& \cdot \int_{0}^{1}(1-u)^{a+p-1} u^{\rho+\delta n} \mathrm{~d} u .
\end{aligned}
$$

$$
\begin{aligned}
\left(\mathscr{F}_{0+}^{a, c, d}\left[\tau^{\rho} \mathcal{F}_{\nu, \eta, \rho, \gamma}^{\mu, \xi, m, \sigma}\left(\tau^{\delta}\right)\right]\right)(s)= & \frac{s^{\rho-c} \Gamma(\rho)}{\Gamma(\eta) \Gamma(\gamma)} \\
& \left.\times{ }_{5} \psi_{4}\left[\begin{array}{l}
(\eta, \xi)(\gamma, \sigma)(\rho+1, \delta)(\rho+d-c+1, \delta)(1,1) \\
(\nu+1, \mu)(\rho, m)(\rho-c+1, \delta)(a+\rho+d+1, \delta)
\end{array}\right)-s^{\delta}\right] .
\end{aligned}
$$

By using equations (11) and (12) in equation (46), we get

$$
\begin{aligned}
& \left(\mathscr{F}_{0+}^{a, c, d}\left[\tau^{\rho} \mathscr{J}_{\nu, \eta, \rho, \gamma}^{\mu, \xi, m, \sigma}\left(\tau^{\delta}\right)\right]\right)(s) \\
& =s^{\rho-c}\left[\begin{array}{l}
\gamma, \sigma \\
\mu, \nu
\end{array} Q_{\rho, m ; n}^{\eta, \xi}\right] \sum_{p=0}^{\infty} \frac{(a+c)_{p}(-d)_{p}}{(a)_{p} p !} \\
& \frac{\left(s^{\delta}\right)^{n} \Gamma(\rho+\delta n+1) \Gamma(a+p)}{\Gamma(a) \Gamma(\rho+\delta n+a+p+1)} .
\end{aligned}
$$
have

By using equations (10) and (16) in equation (47), we

$$
\begin{aligned}
\left(\mathscr{F}_{0+}^{a, c, d}\left[\tau^{\rho} \mathcal{J}_{\nu, \eta, \rho, \gamma}^{\mu, \xi, m, \sigma}\left(\tau^{\delta}\right)\right]\right)(s) \\
=s^{\rho-c}\left[\begin{array}{l}
\gamma, \sigma \\
\mu, \nu \\
{ }_{\rho, m ; n}^{\eta, \xi}
\end{array}\right]\left(s^{\delta}\right)^{n} \\
\\
\quad \cdot \frac{\Gamma(\rho+\delta n+d-c+1) \Gamma(\rho+\delta n+1)}{\Gamma(\rho+\delta n-c+1) \Gamma(\rho+\delta n+a+d+1)} .
\end{aligned}
$$

By using equations (10) and (20) in equation (51), we get

$$
\begin{aligned}
\left(\mathscr{F}_{0+}^{a, c, d}\left[\tau^{\rho} \mathcal{F}_{\nu, \eta, \rho, \gamma}^{\mu, \xi, m, \sigma}\left(\tau^{\delta}\right)\right]\right)(s) \\
=\sum_{n=0}^{\infty} \frac{s^{\rho-c} \Gamma(\rho) \Gamma(\eta+\xi n)}{\Gamma(\eta) \Gamma(\mu n+v+1)} \\
\quad \cdot \frac{\Gamma(\rho+\delta n+d-c+1) \Gamma(\rho+\delta n+1) \Gamma(\gamma+\sigma n)\left(-s^{\delta}\right)^{n}}{\Gamma(\gamma) \Gamma(\rho+m n) \Gamma(\rho+\delta n-c+1) \Gamma(\rho+\delta n+a+d+1)} .
\end{aligned}
$$

Hence, we attain the required result:
Theorem 3. Let $a, c, d, \mu, v, \eta, \rho, \gamma \in \mathbb{C}$ with $\mathfrak{R}(a)>0$, $\rho>\max [0, \mathfrak{R}(c-d)], \quad \mathfrak{R}(\mu)>0, \quad \mathfrak{R}(\nu) \geq-1, \quad \mathfrak{R}(\eta)>0$,

$$
\begin{aligned}
\left(\mathscr{F}_{0-}^{a, c, d}\left[\tau^{-\rho} \mathcal{F}_{\nu, \eta, \rho, \gamma}^{\mu, \xi, m, \sigma}\left(\tau^{-\delta}\right)\right]\right)(s)= & \frac{s^{-c-\rho} \Gamma(\rho)}{\Gamma(\eta) \Gamma(\gamma)} \\
& \left.\times{ }_{5} \psi_{4}\left[\begin{array}{l}
(\eta, \xi)(\gamma, \sigma)(\rho+d, \delta)(\rho+c, \delta)(1,1) \\
(\nu+1, \mu)(\rho, m)(\rho, \delta)(a+\rho+d+c, \delta)
\end{array}\right)-s^{-\delta}\right] .
\end{aligned}
$$


Proof. Consider the right-sided Sagio fractional integral operator (5), in which using the power function with generalized Bessel-Maitland function (19), we get

$$
\mathscr{F}_{0-}^{a, c, d}\left[\tau^{-\rho} \mathscr{J}_{\nu, \eta, \rho, \gamma}^{\mu, \xi, m, \sigma}\left(\tau^{-\delta}\right)\right](s)=\frac{1}{\Gamma(a)} \int_{s}^{\infty} \frac{(\tau-s)^{a-1}}{\tau^{a+c}}{ }_{2} R_{1}\left(a+c,-d ; a ;\left(1-\frac{s}{\tau}\right)\right)\left[\sum_{n=0}^{\infty} \frac{\tau^{-\rho}(\eta)_{\xi n}(\gamma)_{\sigma n}\left(-\tau^{-\delta}\right)^{n}}{\Gamma(\mu n+\nu+1)(\rho)_{m n}}\right] \mathrm{d} \tau
$$

By using equation (8) in equation (52), we have

$$
\begin{aligned}
\mathscr{F}_{0-}^{a, c, d}\left[\tau^{-\rho} \mathcal{J}_{\nu, \eta, \rho, \gamma}^{\mu, \xi, m, \sigma}\left(\tau^{-\delta}\right)\right](s) & =\left[\begin{array}{l}
\gamma, \sigma \\
\mu, \nu
\end{array} Q_{\rho, m ; n}^{\eta, \xi}\right] \int_{s}^{\infty}\left(1-\frac{s}{\tau}\right)^{a-1} \sum_{p=0}^{\infty} \frac{(a+c)_{p}(-d)_{p}(1-(s / \tau))^{p}}{\tau^{c+1} \Gamma(a)(a)_{p} p ! \tau^{\rho}\left(\tau^{\delta}\right)^{n}} \mathrm{~d} \tau \\
& =\left[\begin{array}{l}
\gamma, \sigma \\
\mu, \nu
\end{array} Q_{\rho, m ; n}^{\eta, \xi}\right] \sum_{p=0}^{\infty} \frac{(a+c)_{p}(-d)_{p}}{\Gamma(a)(a)_{p} p !} \int_{s}^{\infty} \frac{(1-(s / \tau))^{a+p-1}}{(\tau)^{\rho+\delta n}} \tau^{-c-1} \mathrm{~d} \tau .
\end{aligned}
$$

By putting the values $(s / \tau)=u \Rightarrow \mathrm{d} \tau=\left(-s / u^{2}\right) \mathrm{d} u$, $\tau=s \Rightarrow u=1$, and $\tau=\infty \Rightarrow u=0$ in equation (53), we obtain

$$
\begin{aligned}
\mathscr{F}_{0-}^{a, c, d}\left[\tau^{-\rho} \mathcal{F}_{\nu, \eta, \rho, \gamma}^{\mu, \xi, m, \sigma}\left(\tau^{-\delta}\right)\right](s) & =\frac{\left[\begin{array}{l}
\gamma, \sigma \\
\mu, \nu
\end{array} Q_{\rho, m ; n}^{\eta, \xi}\right]}{\Gamma(a)} \sum_{p=0}^{\infty} \frac{(a+c)_{p}(-d)_{p}}{(a)_{p} p !} \int_{1}^{0} \frac{(1-u)^{a+p-1}}{(s / u)^{\rho+\delta n+c+1}}\left(\frac{-s}{u^{2}}\right) \mathrm{d} u \\
& =\left[\begin{array}{l}
\gamma, \sigma \\
\mu, \nu
\end{array} Q_{\rho, m ; n}^{\eta, \xi}\right] s^{-c} \sum_{p=0}^{\infty} \frac{(a+c)_{p}(-d)_{p}}{s^{\rho} \Gamma(a)\left((a)_{p} p ! s^{\delta n}\right.} \int_{0}^{1} \frac{(1-u)^{a+p-1}}{u^{-\rho-\delta n-c+1}} \mathrm{~d} u .
\end{aligned}
$$
have

By using equations (11) and (12) in equation (54), we

$$
\begin{aligned}
& \mathscr{F}_{0-}^{a, c, d}\left[\tau^{-\rho} \mathscr{F}_{\nu, \eta, \rho, \gamma}^{\mu, \xi, m, \sigma}\left(\tau^{-\delta}\right)\right](s) \\
& \quad=\left[\begin{array}{l}
\gamma, \sigma \\
\mu, \nu
\end{array} Q_{\rho, m ; n}^{\eta, \xi} s^{-\delta n}\right] s^{-\rho-c} \sum_{p=0}^{\infty} \frac{(a+c)_{p}(-d)_{p} \Gamma(\rho+\delta n+c) \Gamma(a+p)}{\Gamma(a)(a)_{p} p ! \Gamma(\rho+\delta n+a+p+c)} .
\end{aligned}
$$

$$
\begin{aligned}
\mathscr{F}_{0-}^{a, c, d}\left[\tau^{-\rho} \mathcal{J}_{\nu, \eta, \rho, \gamma}^{\mu, \xi, m, \sigma}\left(\tau^{-\delta}\right)\right](s) \\
=\frac{\left[\begin{array}{l}
\gamma, \sigma \\
\mu, \nu
\end{array} Q_{\rho, m ; n}^{\eta, \xi} s^{-\delta n}\right] \Gamma(\rho+\delta n+d) \Gamma(\rho+\delta n+c)}{s^{\rho+c} \Gamma(\rho+\delta n) \Gamma(\rho+\delta n+a+d+c)} .
\end{aligned}
$$
result:

By using equations (10) and (20) in (57), we have the

Now, by using equations (10) and (16) in equation (56), we get

$$
\begin{aligned}
\mathscr{F}_{0-}^{a, c, d}\left[\tau^{-\rho} \mathscr{J}_{\nu, \eta, \rho, \gamma}^{\mu, \xi, m, \sigma}\left(\tau^{-\delta}\right)\right](s) \\
\quad=\sum_{n=0}^{\infty} \frac{s^{-\rho-c} \Gamma(\eta+\xi n)}{\Gamma(\mu n+\nu+1)} \frac{\Gamma(\rho) \Gamma(\gamma+\sigma n) \Gamma(\rho+\delta n+d) \Gamma(\rho+\delta n+c)\left(-s^{-\delta}\right)^{n}}{\Gamma(\eta) \Gamma(\gamma) \Gamma(\rho+m n) \Gamma(\rho+\delta n) \Gamma(\rho+\delta n+a+d+c)} \\
=\frac{s^{-c-\rho} \Gamma(\rho)}{\Gamma(\eta) \Gamma(\gamma)^{5}} \psi_{4}\left[\begin{array}{l}
(\eta, \xi)(\gamma, \sigma)(\rho+d, \delta)(\rho+c, \delta)(1,1) \\
(\nu+1, \mu)(\rho, m)(\rho, \delta)(a+\rho+d+c, \delta)
\end{array} \mid-s^{-\delta}\right] .
\end{aligned}
$$


Theorem 4. Let $\mathfrak{R}(\lambda)>0, \quad \mu, \nu, \eta, \rho, \gamma, w \in \mathbb{C}, \quad \mathfrak{R}(\mu)>0$, $\mathfrak{R}(\nu) \geq-1, \mathfrak{R}(\eta)>0, \mathfrak{R}(\rho)>0, \mathfrak{R}(\gamma)>0, \xi, m, \sigma \geq 0$, and $m, \xi>\Re(\mu)+\sigma$; then, the following relation holds:

$$
\mathscr{F}_{a+}^{\lambda}\left[\frac{\mathscr{F}_{\nu, \eta, \rho, \gamma}^{\mu, \xi, m, \sigma}\left(w(\tau-a)^{\mu}\right)}{(\tau-a)^{-\nu}}\right](s-a)=\frac{\mathscr{J}_{\nu+\lambda, \eta, \rho, \gamma}^{\mu, \xi, m, \sigma}\left(w(s-a)^{\mu}\right)}{(s-a)^{-\lambda-\gamma}} .
$$

Proof. Consider the left-sided Riemann-Liouville fractional integral operator (6), in which using the power function with generalized Bessel-Maitland function (19), we get

$$
\begin{aligned}
& \mathscr{F}_{a+}^{\lambda}\left[(\tau-a)^{\nu} \mathcal{J}_{\nu, \eta, \rho, \gamma}^{\mu, \xi, m, \sigma}\left(w(\tau-a)^{\mu}\right)\right](s-a) \\
& =\left[\begin{array}{l}
\gamma, \sigma \\
\mu, \nu
\end{array} Q_{\rho, m ; n}^{\eta, \xi}\right] \int_{a}^{s} \frac{(\tau-a)^{\nu}\left(w(\tau-a)^{\mu}\right)^{n}}{\Gamma(\lambda)(s-\tau)^{-\lambda+1}} \mathrm{~d} \tau \\
& =\frac{\left[\begin{array}{l}
\gamma, \sigma \\
\mu, \nu
\end{array} Q_{\rho, m ; n}^{\eta, \xi}\right.}{\Gamma(\lambda)(w)^{-n}} \int_{a}^{s}(s-\tau)^{\lambda-1}(\tau-a)^{\nu+\mu n} \mathrm{~d} \tau .
\end{aligned}
$$

By putting the values $((\tau-a) /(s-a))=u \Rightarrow \mathrm{d} \tau=$ $(s-a) \mathrm{d} u, \tau=s \Rightarrow u=1$, and $\tau=a \Rightarrow u=0$ in equation (59), we obtain

$$
\begin{aligned}
& \mathscr{F}_{a+}^{\lambda}\left[(\tau-a)^{\nu} \mathcal{F}_{\nu, \eta, \rho, \gamma}^{\mu, \xi, m, \sigma}\left(w(\tau-a)^{\mu}\right)\right](s-a) \\
& =\frac{\left[\begin{array}{l}
\gamma, \sigma \\
\mu, \nu
\end{array} Q_{\rho, m ; n}^{\eta, \xi}\right](w)^{n}}{\Gamma(\lambda)} \int_{0}^{1} \frac{(s-(s-a) u-a)^{\lambda-1}}{((s-a) u)^{-\mu n-\nu}(s-a)^{-1}} \mathrm{~d} u \\
& =\frac{(s-a)^{\lambda+v}}{(w)^{-n} \Gamma(\lambda)}\left[{ }_{\mu, \nu}^{\gamma, \sigma} Q_{\rho, m ; n}^{\eta, \xi}(s-a)^{\mu n}\right] \int_{0}^{1}(1-u)^{\lambda-1} u^{\mu n+v} \mathrm{~d} u .
\end{aligned}
$$
have

By using equations (11) and (12) in equation (60), we

$$
\begin{aligned}
\mathscr{F}_{a+}^{\lambda} & {\left[(\tau-a)^{\nu} \mathcal{J}_{\nu, \eta, \rho, \gamma}^{\mu, \xi, m, \sigma}\left(w(\tau-a)^{\mu}\right)\right](s-a) } \\
& \left.=(s-a)^{\lambda+v}{ }_{\mu, \nu}^{\gamma, \sigma} Q_{\rho, m ; n}^{\eta, \xi}(w)^{n}(s-a)^{\mu n}\right] \frac{\Gamma(\mu n+v+1)}{\Gamma(\lambda+\mu n+\nu+1)} .
\end{aligned}
$$

Now, by using equation (20) in equation (61), then the required result is obtained:

$$
\begin{aligned}
\mathscr{F}_{a+}^{\lambda} & {\left[(\tau-a)^{\nu} \mathcal{F}_{\nu, \eta, \rho, \gamma}^{\mu, \xi, m, \sigma}\left(w(\tau-a)^{\mu}\right)\right](s-a) } \\
= & (s-a)^{\lambda+v} \sum_{n=0}^{\infty} \frac{(\eta)_{\xi n}(\gamma)_{\sigma n}\left(-w(s-a)^{\mu}\right)^{n}}{\Gamma(\lambda+\mu n+v+1)(\rho)_{m n}} \\
= & (s-a)^{\lambda+v} \mathcal{F}_{\nu+\lambda, \eta, \rho, \gamma}^{\mu, \xi, m, \sigma}\left(w(s-a)^{\mu}\right) .
\end{aligned}
$$

\section{Riemann-Liouville Fractional Operators and Laplace Transform of the New Operator}

In this section, we discuss the Riemann-Liouville fractional integral and differential operators with the fractional integral operator. Also, we developed a result which deals with the Laplace transform of the new fractional integral operator.

Theorem 5. Let $w, \lambda, \mu, \nu, \eta, w, \gamma \in \mathbb{C}, \mathfrak{R}(\alpha)>0, \mathfrak{R}(\beta)>0$, $\mathfrak{R}(\lambda)>0, \quad \mathfrak{R}(\mu)>0, \quad \mathfrak{R}(\nu) \geq-1, \quad \mathfrak{R}(\eta)>0, \quad \mathfrak{R}(w)>0$, $\mathfrak{R}(\gamma)>0, \xi, m, \sigma \geq 0, \mathfrak{R}(\rho)>0$, and $m, \xi>\mathfrak{R}(\mu)+\sigma$; then, the following relation holds:

$$
\left(\mathscr{F}_{0^{+}}^{\lambda} \mathscr{Z}_{\nu, \eta, \rho, \gamma, w, 0^{+}}^{\mu, \xi, m, \sigma} \phi\right)(s)=\left(\mathscr{Z}_{\nu+\lambda, \eta, \rho, \gamma, w, 0^{+}}^{\mu, \xi, m, \sigma} \phi\right)(s) .
$$

Proof. Consider the left-sided Riemann-Liouville integral operator (6) involving new fractional integral operator (22) as

$$
\begin{aligned}
& \left(\mathscr{F}_{0^{+}}^{\lambda} \mathscr{Z}_{\nu, \eta, \rho, \gamma, \gamma, w, 0^{+}}^{\mu, \xi} \phi\right)(s) \\
& \quad=\frac{1}{\Gamma(\lambda)} \int_{0}^{s}(s-u)^{\lambda-1} \int_{0}^{u}(u-\tau)^{\nu} \mathscr{F}_{\nu, \eta, \rho, \gamma}^{\mu, \xi, m, \sigma}\left(w(u-\tau)^{\mu}\right) \phi(\tau) \mathrm{d} \tau \mathrm{d} u .
\end{aligned}
$$

By using equation (18) in equation (63), we have

$$
\begin{aligned}
& \left(\mathscr{F}_{0^{+}}^{\lambda} \mathscr{Z}_{\nu, \eta, \rho, \gamma, w, 0^{+}}^{\mu, \xi, m, \sigma} \phi\right)(s) \\
& \quad=\frac{1}{\Gamma(\lambda)} \int_{0}^{s} \int_{\tau}^{s}(s-u)^{\lambda-1}(u-\tau)^{\nu} \mathcal{F}_{\nu, \eta, \rho, \gamma}^{\mu, \xi, m, \sigma}\left(w(u-\tau)^{\mu}\right) \mathrm{d} u \phi(\tau) \mathrm{d} \tau .
\end{aligned}
$$

By putting the values $t=u-\tau \Rightarrow \mathrm{d} t=\mathrm{d} u, u=s \Rightarrow t=$ $s-\tau$, and $u=\tau \Rightarrow t=0$ in equation (64), we get

$$
\begin{aligned}
\left(\mathscr{F}_{0^{+}}^{\lambda} \mathscr{Z}_{\nu, \eta, \rho, \gamma, w, 0^{+}}^{\mu, \xi, m)}(s)\right. & =\frac{1}{\Gamma(\lambda)} \int_{0}^{s} \int_{0}^{s-\tau}(t)^{\nu} \frac{\mathcal{F}_{\nu, \eta, j, \gamma, \gamma}^{\mu, \xi, m, \sigma}\left(w(t)^{\mu}\right)}{(s-\tau-t)^{1-\lambda}} \mathrm{d} t \phi(\tau) \mathrm{d} \tau \\
& =\int_{0}^{s} \frac{1}{\Gamma(\lambda)} \int_{0}^{s-\tau}(t)^{\nu} \frac{\mathcal{F}_{\nu, \eta, \rho, \gamma}^{\mu, \xi, m, \sigma}\left(w(t)^{\mu}\right)}{(s-\tau-t)^{1-\lambda}} \mathrm{d} t \phi(\tau) \mathrm{d} \tau .
\end{aligned}
$$

By using equation (6) in equation (65), we have

$$
\begin{aligned}
\left(\mathscr{F}_{0^{+}}^{\lambda} \mathscr{Z}_{\nu, \eta, \rho, \gamma, w, 0^{+}}^{\mu, \xi, m, \sigma} \phi\right)(s)= & \int_{0}^{s}\left[\mathscr{F}_{0^{+}}^{\lambda}(t)^{\nu} \mathscr{F}_{\nu, \eta, \rho, \gamma}^{\mu, \xi, m, \sigma}\left(w(t)^{\mu}\right)\right] \\
& \cdot(s-\tau) \phi(\tau) \mathrm{d} \tau .
\end{aligned}
$$

By using equation (58) in equation (66), we obtain

$$
\begin{aligned}
\left(\mathscr{F}_{0^{+}}^{\lambda} \mathscr{Z}_{\nu, \eta, \rho, \gamma, w, 0^{+}}^{\mu, \xi, m, \sigma} \phi\right)(s) & =\int_{0}^{s} \frac{\mathscr{J}_{\nu+\lambda, \eta, \rho, \gamma}^{\mu, \xi, m, \sigma}\left(w(s-\tau)^{\mu}\right)}{(s-\tau)^{-\nu-\lambda}} \phi(\tau) \mathrm{d} \tau \\
& =\left(\mathscr{Z}_{\nu+\lambda, \eta, \rho, \gamma, w, 0^{+}}^{\mu, \xi, m, \sigma}\right)(s) .
\end{aligned}
$$

Theorem 6. Let $\lambda, \mu, \nu, \eta, w, \gamma \in \mathbb{C}, \quad \Re(\alpha)>0, \mathfrak{R}(\beta)>0$, $\mathfrak{R}(\lambda)>0, \quad \mathfrak{R}(\mu)>0, \quad \mathfrak{R}(\nu) \geq-1, \quad \mathfrak{R}(\eta)>0, \quad \mathfrak{R}(w)>0$, 
$\mathfrak{R}(\gamma)>0, \xi, m, \sigma \geq 0, \mathfrak{R}(\rho)>0$, and $m, \xi>\mathfrak{R}(\mu)+\sigma$; then, the following relation holds:

$$
\left(\mathscr{D}_{0^{+}}^{\lambda} \mathscr{Z}_{\nu, \eta, \rho, \gamma, w, 0^{+}}^{\mu, \xi} \phi\right)(s)=\left(\mathscr{Z}_{\nu-\lambda, \eta, \rho, \gamma, w, 0^{+}}^{\mu, \xi, m, \sigma} \phi\right)(s) .
$$

Proof. Consider the left-sided Riemann-Liouville differential operator (7) involving new fractional integral operator (22); then,

$$
\begin{aligned}
\left(\mathscr{D}_{0^{+}}^{\lambda} \mathscr{Z}_{\nu, \eta, \rho, \gamma, w, 0^{+}}^{\mu, \xi, m, \sigma} \phi\right)(s) \\
=\frac{1}{\Gamma(m-\lambda)}\left(\frac{\mathrm{d}}{\mathrm{d} s}\right)^{m} \int_{0}^{s}(s-u)^{m-\lambda-1} \\
\quad \cdot \int_{0}^{u} \frac{\mathcal{F}^{\mu, \xi, \eta, m, \sigma, \gamma}\left(w(u-\tau)^{\mu}\right)}{(u-\tau)^{-\nu}} \phi(\tau) \mathrm{d} \tau \mathrm{d} u .
\end{aligned}
$$

By using equation (18) in equation (69), we have

$$
\begin{aligned}
& \left(\mathscr{D}_{0^{+}}^{\lambda} \mathscr{Z}_{\nu, \eta, \rho, \gamma, w, 0^{+}}^{\mu, \xi, m, \sigma}\right)(s) \\
& =\frac{1}{\Gamma(m-\lambda)}\left(\frac{\mathrm{d}}{\mathrm{d} s}\right)^{m} \int_{0}^{s} \int_{\tau}^{s}(\tau-u)^{\nu} \frac{\mathscr{J}_{\nu, \eta, \rho, \gamma}^{\mu, \xi, m, \sigma}\left(w(u-\tau)^{\mu}\right)}{(s-u)^{\lambda-m+1}} \mathrm{~d} u \phi(\tau) \mathrm{d} \tau .
\end{aligned}
$$

By putting the values $t=u-\tau \Rightarrow \mathrm{d} t=\mathrm{d} u$, $u=s \Rightarrow t=s-\tau$, and $u=\tau \Rightarrow t=0$ in equation (70), we get

$$
\begin{aligned}
& \left(\mathscr{D}_{0^{+}}^{\lambda} \mathscr{Z}_{\nu, \eta, \rho, \gamma, w, 0^{+}}^{\mu, \xi, m, \sigma} \phi\right)(s) \\
& \quad=\frac{1}{\Gamma(m-\lambda)}\left(\frac{\mathrm{d}}{\mathrm{d} s}\right)^{m} \int_{0}^{s} \int_{0}^{s-\tau} \frac{(t)^{\nu} \mathscr{J}_{\nu, \eta, \rho, \gamma}^{\mu, \xi, m, \sigma}\left(w(t)^{\mu}\right)}{(s-\tau-t)^{-m+\lambda+1}} \mathrm{~d} t \phi(\tau) \mathrm{d} \tau \\
& \quad=\int_{0}^{s}\left(\frac{\mathrm{d}}{\mathrm{d} x}\right)^{m} \frac{1}{\Gamma(m-\lambda)} \int_{0}^{s-\tau} \frac{(t)^{\nu} \mathcal{J}_{\nu, \eta, \rho, \gamma}^{\mu, \xi, m, \sigma}\left(w(t)^{\mu}\right)}{(s-\tau-t)^{-m+\lambda+1}} \mathrm{~d} t \phi(\tau) \mathrm{d} \tau .
\end{aligned}
$$

Now, by using equation (6) in equation (71), we have

$$
\begin{aligned}
& \left(\mathscr{D}_{0^{+}}^{\lambda} \mathscr{Z}_{\nu, \eta, \rho, \gamma, w, 0^{+}}^{\mu, \xi} \phi\right)(s) \\
& \quad=\int_{0}^{s}\left(\frac{\mathrm{d}}{\mathrm{d} s}\right)^{m} \mathscr{F}_{0^{+}}^{m-\lambda}\left[(t)^{\nu} \mathscr{J}_{\nu, \eta, \rho, \gamma}^{\mu, \xi, m, \sigma}\left(w(t)^{\mu}\right)\right](s-\tau) \phi(\tau) \mathrm{d} \tau .
\end{aligned}
$$

By using equation (58) in equation (72), we obtain

$$
\begin{aligned}
& \left(\mathscr{D}_{0^{+}}^{\lambda} \mathscr{Z}_{\nu, \eta, \rho, \gamma, w, 0^{+}}^{\mu, \xi, m, \sigma} \phi\right)(s) \\
& \quad=\int_{0}^{s}\left(\frac{\mathrm{d}}{\mathrm{d} s}\right)^{m} \frac{\mathscr{J}_{m+\nu-\lambda, \eta, \rho, \gamma}^{\mu, \xi, m, \sigma}\left(w(s-\tau)^{\mu}\right)}{(s-\tau)^{\lambda-m-\nu}} \phi(\tau) \mathrm{d} \tau .
\end{aligned}
$$

By using equation (19) in equation (73) and then taking one-time derivative, we have

$$
\begin{aligned}
& \left(\mathscr{D}_{0^{+}}^{\lambda} \mathscr{Z}_{\nu, \eta, \rho, \gamma, w, 0^{+}}^{\mu, \xi, m, \sigma} \phi\right)(s)=\sum_{n=0}^{\infty} \frac{(-w)^{n}(\mu n+m+\nu-\lambda)}{\Gamma(\mu n+m+\nu-\lambda+1)} \times \frac{(\eta)_{\xi n}(\gamma)_{\sigma n}}{(w)_{m n}}\left(\frac{\mathrm{d}}{\mathrm{d} s}\right)^{m-1} \int_{0}^{s}(s-\tau)^{-\lambda+\mu n+m+\nu-1} \phi(\tau) \mathrm{d} \tau \\
& =\left(\frac{\mathrm{d}}{\mathrm{d} s}\right)^{m-1} \int_{0}^{s} \frac{\mathcal{J}_{\nu+m-\lambda-1, \eta, \rho, \gamma}^{\mu, m, \sigma}\left(w(s-\tau)^{\mu}\right)}{(s-\tau)^{-m-\nu+\lambda+1}} \phi(\tau) \mathrm{d} \tau
\end{aligned}
$$

Now, taking the $(m-1)$ derivative of equation (74), we get

$$
\begin{aligned}
\left(\mathscr{D}_{0^{+}}^{\lambda} \mathscr{E}_{\nu, \eta, \rho, \gamma, w, 0^{+}}^{\mu, \xi, m, \sigma} \phi\right)(s) & =\int_{0}^{s} \frac{\mathcal{F}^{\mu, \xi, m, \eta, \eta, \rho, \gamma}\left(w(s-\tau)^{\mu}\right)}{(s-\tau)^{\lambda-\gamma}} \phi(\tau) \mathrm{d} \tau \\
& =\left(\mathscr{Z}_{\nu-\lambda, \eta, \rho, \gamma, w, 0^{+}}^{\mu, \xi, m, \sigma} \phi\right)(s) .
\end{aligned}
$$

Theorem 7. Let $\quad w, \dot{v}, \dot{\eta}, \hat{\gamma}, \mu, v, \eta, \gamma \in \mathbb{C} \in \mathbb{C}, \quad \mathfrak{R}(\eta)>0$, $\mathfrak{R}(\gamma)>0, \mathfrak{R}(\mu)>0, \mathfrak{R}(\nu) \geq-1, \mathfrak{R}(\eta)>0, \mathfrak{R}(w)>0$, and $\mathfrak{R}(\gamma)>0$; then, the following relation holds:

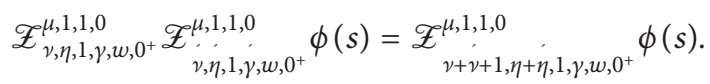

Proof. Consider the new fractional integral operator (22):

$$
\begin{aligned}
& \mathscr{Z}_{\nu, \eta, 1, \gamma, w, 0^{+}}^{\mu, 1,1,0} \mathscr{Z}_{\nu, \eta, 1, \gamma, w, 0^{+}}^{\mu, 1,1,0} \phi(s) \\
& =\int_{a}^{s} \frac{\mathcal{J}_{\nu, \eta, 1, \gamma}^{\mu, 1,1,0}\left(w(s-u)^{\mu}\right)}{(s-u)^{-\nu}} \mathrm{d} u \int_{a}^{u} \frac{\mathcal{F}^{\mu, 1,1,0},\left(w(u-t)^{\mu}\right)}{(u-t)^{-\nu}} \phi(t) \mathrm{d} t \\
& =\int_{a}^{s} \phi(t) \mathrm{d} t \int_{t}^{s} \frac{\mathscr{J}_{\nu, \eta, 1, \gamma}^{\mu, 1,1,0}\left(w(s-u)^{\mu}\right) \mathcal{F}_{\nu, \eta, 1, \gamma}^{\mu, 1,1,0}\left(w(u-t)^{\mu}\right)}{(s-u)^{-\nu}(u-t)^{-\gamma}} \mathrm{d} u .
\end{aligned}
$$

By reversing the order of integration and putting $\tau=((s-u) /(s-t))$, the inner integral is 


$$
\begin{aligned}
& \mathscr{Z}_{\nu, \eta, 1, \gamma, w, 0^{+}}^{\mu, 1,1,0} \mathscr{Z}_{\nu, \eta, 1,1, \gamma, w, 0^{+}}^{\mu, 1,1,0} \phi(s) \\
& =\int_{0}^{1} \frac{\mathcal{J}_{\nu, \eta, 1, \gamma}^{\mu, 1,1,0}(w(s-t) \tau)^{\mu} \mathcal{J}_{\nu, \eta, 1, \gamma}^{\mu, 1,1, \sigma}\left(w(s-(s-t) \tau-t)^{\mu}\right)}{((s-t) \tau)^{-\nu}(s-(s-t) \tau-t)^{-\nu}(s-t)^{-1}} \mathrm{~d} \tau \\
& =\sum_{p=0}^{\infty} \frac{(\eta)_{p}(-w)^{p}(s-t)^{\mu p}}{\Gamma(\mu p+\nu+1) p !} \sum_{n=0}^{\infty} \frac{(\eta)_{n}(-w)^{n}(s-t)^{\mu n}}{\Gamma(\mu n+\dot{v}+1) n !} \\
& \times(s-t)^{\nu+\nu+1} \int_{0}^{1} \tau^{\nu+\mu p}(1-\tau)^{\nu+\mu n} \mathrm{~d} \tau \\
& =(s-t)^{\nu+\nu+1} \sum_{n=0}^{\infty} \sum_{p=0}^{\infty} \frac{(\eta)_{p}(-w)^{p+n}(s-t)^{\mu(p+n)}(\eta)_{n}}{\Gamma(\mu p+\mu n+\nu+\dot{v}+1+1) p ! n !} \\
& =(s-t)^{\nu+\nu+1} \sum_{p=0}^{\infty} \frac{(-w)^{p}(s-t)^{\mu p}}{\Gamma(\mu p+\nu+\nu+1+1)} \sum_{n=0}^{\infty} \frac{(\eta)_{p-n}(\eta)_{n}}{(p-n) ! n !} .
\end{aligned}
$$

This implies that

$$
\begin{aligned}
& \mathscr{Z}_{\nu, \eta, 1, \gamma, w, 0^{+}}^{\mu, 1,1,0} \mathscr{Z}_{\nu, \eta, 1, \gamma, w, 0^{+}}^{\mu, 1,1,0} \phi(s)
\end{aligned}
$$

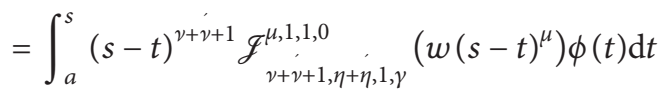

$$
\begin{aligned}
& =\mathscr{Z}^{\mu, 1,1,0}, \quad \phi(s) .
\end{aligned}
$$

Theorem 8. Let $\mu, v, \eta, \rho, \gamma, w \in \mathbb{C}, \mathfrak{R}(\mu)>0, \mathfrak{R}(\nu) \geq-1$, $\mathfrak{R}(\eta)>0, \quad \mathfrak{R}(\rho)>0, \quad \mathfrak{R}(\gamma)>0, \quad \xi, m, \sigma \geq 0, \quad$ and $m, \xi>\Re(\mu)+\sigma$; then, the following relation holds:

$$
\begin{aligned}
& \mathscr{L}\left[\mathscr{Z}_{\nu, \eta, \rho, \gamma, w, 0^{+}}^{\mu, \xi} \phi\right] \\
& \quad=\frac{s^{-v-1} \Gamma(\rho)}{\Gamma(\eta) \Gamma(\gamma)^{4}} \psi_{2}\left[\begin{array}{l}
(\eta, \xi)(\gamma, \sigma)(\nu, \mu)(1,1) \\
(\nu+1, \mu)(\rho, m)
\end{array} \mid-\left(\frac{w}{s}\right)^{\mu}\right] .
\end{aligned}
$$

Proof. Consider fractional integral operator (22):

$$
\mathscr{L}\left[\mathscr{Z}_{\nu, \eta, \rho, \gamma, w, 0^{+}}^{\mu, \xi, m, \sigma} \phi\right]=\int_{0}^{\infty} e^{-s t}\left[\int_{0}^{t}(t-u)^{\nu} \sum_{n=0}^{\infty} \frac{(\eta)_{\xi n}(\gamma)_{\sigma n}(-w)^{n}(t-u)^{\mu n}}{\Gamma(\mu n+\nu+1)(\rho)_{m n}} \phi(u) \mathrm{d} u\right] \mathrm{d} t .
$$

Now, after changing the order of integration, we obtain

$$
\begin{aligned}
\mathscr{L}\left[\mathscr{Z}_{v, \eta, \rho, \gamma, w, 0^{+}}^{\mu, \xi, m, \sigma} \phi\right] & =\int_{0}^{\infty} \int_{u}^{\infty} \frac{(t-u)^{\nu}}{e^{s t}} \sum_{n=0}^{\infty} \frac{(\eta)_{\xi n}(\gamma)_{\sigma n}(-w)^{n}(t-u)^{\mu n}}{\Gamma(\mu n+\nu+1)(\rho)_{m n}} \mathrm{~d} t \phi(u) \mathrm{d} u \\
& =\sum_{n=0}^{\infty} \frac{(\eta)_{\xi n}(\gamma)_{\sigma n}(-w)^{n}}{\Gamma(\mu n+\nu+1)(\rho)_{m n}} \int_{0}^{\infty} \int_{u}^{\infty} \frac{(t-u)^{\mu n+\nu}}{e^{s t}} \mathrm{~d} t \phi(u) \mathrm{d} u .
\end{aligned}
$$

By putting $t-u=\tau$, then

$$
\begin{aligned}
\mathscr{L}\left[\mathscr{Z}_{\nu, \eta, \rho, \gamma, w, 0^{+}}^{\mu, \xi, m, \sigma} \phi\right] & =\sum_{n=0}^{\infty} \frac{(\eta)_{\xi n}(\gamma)_{\sigma n}(-w)^{n}}{\Gamma(\mu n+\nu+1)(\rho)_{m n}} \int_{0}^{\infty} \frac{\phi(u)}{e^{s u}} \int_{0}^{\infty} e^{-s \tau} \tau^{\mu n+v} \mathrm{~d} \tau \mathrm{d} u \\
& =\sum_{n=0}^{\infty} \frac{(\eta)_{\xi n}(\gamma)_{\sigma n}(-w)^{n}}{\Gamma(\mu n+\nu+1)(\rho)_{m n}} \frac{\Gamma(\mu n+\nu)}{s^{\mu n+\nu+1}} \phi(s) \\
& =\frac{s^{-v-1} \Gamma(\rho)}{\Gamma(\eta) \Gamma(\gamma)} \sum_{n=0}^{\infty} \frac{\Gamma(\eta+\xi n) \Gamma(\gamma+\sigma n)\left(-w s^{-\mu}\right)^{n} \Gamma(\mu n+\nu)}{\Gamma(\mu n+\nu+1) \Gamma(\rho+m n)} \\
& =\frac{s^{-v-1} \Gamma(\rho)}{\Gamma(\eta) \Gamma(\gamma)^{4}} \psi_{2}\left[\begin{array}{l}
(\eta, \xi)(\gamma, \sigma)(\nu, \mu)(1,1) \\
(\nu+1, \mu)(\rho, m)
\end{array} \mid-\left(\frac{w}{s}\right)^{\mu}\right] .
\end{aligned}
$$


Theorem 9. Let $\lambda, \delta, \chi, \mu, \nu, \eta, \rho, \gamma, w \in \mathbb{C}, \quad \mathfrak{R}(\mu)>0$, $\mathfrak{R}(\nu) \geq-1, \mathfrak{R}(\eta)>0, \mathfrak{R}(\rho)>0, \mathfrak{R}(\gamma)>0, \xi, m, \sigma \geq 0$, and $m, \xi>\Re(\mu)+\sigma$; then, the following relation holds:

$$
\left(\mathscr{Z}_{\nu, \eta, \rho, \gamma, w, 0^{+}}^{\mu, \xi} t^{((\lambda+\delta) / \chi)-1}\right)(s)=\frac{s^{\nu+((\lambda+\delta) / \chi)} \Gamma(\rho) \Gamma((\lambda+\delta) / \chi)}{\Gamma(\eta) \Gamma(\gamma)}{ }_{3} \psi_{2}\left[\begin{array}{c}
(\eta, \xi)(\gamma, \sigma)(1,1) \\
\left(\nu+\frac{\lambda+\delta}{\chi}+1, \mu\right)(\rho, m)
\end{array} \mid-w s^{\mu}\right] .
$$

Proof. Consider the new fractional integral operator (22):

$$
\begin{aligned}
\left(\mathscr{Z}_{\nu, \eta, \rho, \gamma, w, 0^{+}}^{\mu, \xi, m, \sigma} t^{((\lambda+\delta) / \chi)-1}\right)(s) & =\int_{0}^{s}(s-\tau)^{\nu} \mathcal{J}_{\nu, \eta, \rho, \gamma}^{\mu, \xi, m, \sigma}\left[w(s-\tau)^{\mu}\right] \tau^{((\lambda+\delta) / \chi)-1} \mathrm{~d} \tau \\
& =\sum_{n=0}^{\infty} \frac{(\eta)_{\xi n}(\gamma)_{\sigma n}(-w)^{n} s^{\mu n+\gamma}}{\Gamma(\mu n+\nu+1)(\rho)_{m n}} \int_{0}^{s}\left(1-\frac{\tau}{s}\right)^{\mu n+\nu} \tau^{((\lambda+\delta) / \chi)-1} \mathrm{~d} \tau .
\end{aligned}
$$

By putting $(\tau / s)=u$, then we have

$$
\begin{aligned}
\left(\mathscr{Z}_{\nu, \eta, \rho, \gamma, w, 0^{+}}^{\mu, \xi, m, \sigma} t^{((\lambda+\delta) / \chi)-1}\right)(s) & =\sum_{n=0}^{\infty} \frac{(\eta)_{\xi n}(\gamma)_{\sigma n}(-w)^{n} s^{\mu n+v}}{\Gamma(\mu n+\nu+1)(\rho)_{m n}} \int_{0}^{s}(1-u)^{\mu n+v}(s u)^{((\lambda+\delta) / \chi)-1} s \mathrm{~d} u \\
& =\sum_{n=0}^{\infty} \frac{(\eta)_{\xi n}(\gamma)_{\sigma n}(-w)^{n} s^{\mu n+\nu+((\lambda+\delta) / \chi)}}{\Gamma(\mu n+\nu+1)(\rho)_{m n}} \frac{\Gamma(\mu n+\nu+1) \Gamma((\lambda+\delta) / \chi)}{\Gamma(\mu n+\nu+((\lambda+\delta) / \chi)+1)} \\
& =\frac{s^{\nu+((\lambda+\delta) / \chi)} \Gamma(\rho)}{\Gamma(\eta) \Gamma(\gamma)} \sum_{n=0}^{\infty} \frac{\Gamma(\eta+\xi n) \Gamma(\gamma+\sigma n)\left(-w s^{\mu}\right)^{n} \Gamma((\lambda+\delta) / \chi)}{\Gamma(\mu n+\nu+((\lambda+\delta) / \chi)+1) \Gamma(\rho+m n)} \\
& =\frac{s^{\nu+((\lambda+\delta) / \chi)} \Gamma(\rho) \Gamma((\lambda+\delta) / \chi)}{\Gamma(\eta) \Gamma(\gamma)}{ }_{3} \psi_{2}\left[\begin{array}{c}
(\eta, \xi)(\gamma, \sigma)(1,1) \\
\left.\nu+\frac{\lambda+\delta}{\chi}+1, \mu\right)(\rho, m) \mid-w s^{\mu}
\end{array}\right]
\end{aligned}
$$

\section{Inverse Operator with Some Special Functions}

In this section, we discuss some applications of the inverse fractional operator. We derive some results of the inverse fractional operator with the Mittag-Leffler function and
Bessel-Maitland function, and results can be seen in the form of Wright functions.

Theorem 10. Consider $\delta, \alpha, \beta, \mu, \nu, \eta, \rho, \gamma, \lambda, \in \mathbb{C}$ with $\min \{\mathfrak{R}(\delta), \mathfrak{R}(\alpha), \mathfrak{R}(\beta)\}>0, p, q>0, \mathfrak{R}(\mu)>0, \mathfrak{R}(\nu) \geq-1$, $\mathfrak{R}(\eta)>0, \quad \mathfrak{R}(\rho)>0, \quad \mathfrak{R}(\gamma)>0, \quad \xi, m, \sigma \geq 0, \quad$ and $m, \xi>\Re(\mu)+\sigma$; then, the following relation holds:

$$
\begin{aligned}
{\left[D_{\nu, \eta, \rho, \gamma, w, a^{+}}^{\mu, \xi, m, \sigma}(\tau-a)^{\rho-1} E_{\alpha, \beta, p}^{\gamma, \delta, q}(\tau-a)^{\lambda}\right](s)=} & \frac{\Gamma(\rho) \Gamma(\delta)}{\Gamma(\gamma) \Gamma(\eta) \Gamma(\gamma)} \sum_{m=0}^{\infty} \frac{\Gamma(\gamma+q m)}{\Gamma(\alpha m+\beta)} \\
& \times(s-a)_{3}^{\lambda m+\rho-v} \psi_{2}\left[\begin{array}{l}
(\eta, \xi)(\gamma, \sigma)(1,1) \\
(\rho, m)(\lambda m+\rho-\nu+1, \mu)
\end{array} \mid-w(s-a)^{\mu}\right] .
\end{aligned}
$$


Proof. Consider inverse fractional integral operator (23) with Mittag-Leffler function (28); then, the following results hold:

$$
\begin{aligned}
& {\left[D_{\nu, \eta, \rho, \gamma, w, a^{+}}^{\mu, \xi, a, \sigma}(\tau-a)^{\rho-1} E_{\alpha, \beta, p}^{\gamma, \delta, q}(\tau-a)^{\lambda}\right](s)} \\
& \quad=\left(\frac{\mathrm{d}}{\mathrm{d} s}\right)^{p} \int_{a}^{s}(s-\tau)^{p-\nu} \sum_{n=0}^{\infty} \frac{(\eta)_{\xi n}(\gamma)_{\sigma n}\left(-w(s-\tau)^{\mu}\right)^{n}}{(\rho)_{m n} \Gamma(\mu n+p-\nu+1)}(\tau-a)^{\rho-1} \sum_{m=0}^{\infty} \frac{(\gamma)_{q m}(\tau-a)^{\lambda m}}{\Gamma(\alpha m+\beta)(\delta)_{p m}} \mathrm{~d} \tau \\
& \quad=\left(\frac{\mathrm{d}^{p}}{\mathrm{~d} s^{p}}\right)_{\mu, p-\nu}^{\gamma, \sigma} Q_{\rho, m ; n}^{\eta, \xi}(w)^{n} \sum_{m=0}^{\infty} \frac{(\gamma)_{q m}}{\Gamma(\alpha m+\beta)(\delta)_{p m}} \int_{a}^{s}(s-\tau)^{\mu n+p-\nu}(\tau-a)^{\rho+\lambda m-1} \mathrm{~d} \tau .
\end{aligned}
$$

Substituting $u=((s-\tau) /(s-a))$ in equation (88), we obtain

$$
\begin{aligned}
& {\left[D_{\nu, \eta, \rho, \gamma, w, a^{+}}^{\mu, \xi, a, \sigma}(\tau-a)^{\rho-1} E_{\alpha, \beta, p}^{\gamma, \delta, q}(\tau-a)^{\lambda}\right](s)} \\
& \quad=\left(\frac{\mathrm{d}^{p}}{\mathrm{~d} s^{p}}\right)_{\mu, p-\gamma}^{\gamma, \sigma} Q_{\rho, m ; n}^{\eta, \xi}(w)^{n} \sum_{m=0}^{\infty} \frac{(\gamma)_{q m}(s-a)^{p-\nu+\mu n+\lambda m+\rho}}{\Gamma(\alpha m+\beta)(\delta)_{p m}} \int_{0}^{1}(1-u)^{\mu n+p-\nu}(u)^{\rho+\lambda m-1} \mathrm{~d} u .
\end{aligned}
$$

By using equations (11) and (12), we get

$$
\begin{aligned}
& {\left[D_{\nu, \eta, \rho, \gamma, w, a^{+}}^{\mu, \xi, m, \sigma}(\tau-a)^{\rho-1} E_{\alpha, \beta, p}^{\gamma, \delta, q}(\tau-a)^{\lambda}\right](s)} \\
& \quad=\left(\frac{\mathrm{d}^{p}}{\mathrm{~d}^{p} p}\right)_{\mu, p-\nu}^{\gamma, \sigma} Q_{\rho, m ; n}^{\eta, \xi}(w)^{n} \sum_{m=0}^{\infty} \frac{(\gamma)_{q m}(s-a)^{p-\nu+\mu n+\lambda m+\rho}}{\Gamma(\alpha m+\beta)(\delta)_{p m}} \frac{\Gamma(\mu n+p-\nu+1) \Gamma(\rho+\lambda m)}{\Gamma(\mu n+p-\nu+\rho+\lambda m+1)} .
\end{aligned}
$$
have

Now, back-substituting ${ }_{\mu, p-\nu}^{\gamma, \sigma} Q_{\rho, m ; n}^{\eta, \xi}$ in equation (90), we

$$
\begin{aligned}
& {\left[D_{\nu, \eta, \rho, \gamma, w, a^{+}}^{\mu, \xi, m, \sigma}(\tau-a)^{\rho-1} E_{\alpha, \beta, p}^{\gamma, \delta, q}(\tau-a)^{\lambda}\right](s)} \\
& =\sum_{m, n=0}^{\infty} \frac{(\eta)_{\xi n}(\gamma)_{\sigma n}\left(\mathrm{~d}^{p} / \mathrm{d} s^{p}\right)(-w)^{n}(s-a)^{p-\gamma+\mu n+\lambda m+\rho}}{(\rho)_{m n} \Gamma(\mu n+\rho+\lambda m+p-\nu+1)} \frac{(\dot{\gamma})_{q m} \Gamma(\rho+\lambda m)}{\Gamma(\alpha m+\beta)(\delta)_{p m}} \\
& =\sum_{m, n=0}^{\infty} \frac{(\eta)_{\xi n}(\gamma)_{\sigma n}(s-a)^{-v+\mu n+\lambda m+\rho}}{(\rho(\mu n+\rho+\lambda m-\nu+1)} \frac{(\gamma)_{q m} \Gamma(\rho+\lambda m)}{\Gamma(\alpha m+\beta)(\delta)_{p m}} \\
& \left.=\frac{\Gamma(\rho) \Gamma(\delta)}{\Gamma(\gamma) \Gamma(\eta) \Gamma(\gamma)} \sum_{m=0}^{\infty} \frac{\Gamma(\gamma+q m)(s-a)^{\lambda m+\rho-v}}{\Gamma(\alpha m+\beta)} \mid-w(s-a)^{\mu}\right] .
\end{aligned}
$$


Corollary 1. On setting $v=-v$ in Theorem 10, we obtain the result in the form of the differential operator:

$$
\begin{aligned}
{\left[\mathscr{Z}_{\nu, \eta, \rho, \gamma, w, a^{+}}^{\mu, \xi, m, \sigma}(\tau-a)^{\rho-1} E_{\alpha, \beta, p}^{\gamma, \delta, q}(\tau-a)^{\lambda}\right](s)=} & \frac{\Gamma(\rho) \Gamma(\delta)}{\Gamma(\gamma) \Gamma(\eta) \Gamma(\dot{\gamma})} \sum_{m=0}^{\infty} \frac{\Gamma(\gamma+q m)}{\Gamma(\alpha m+\beta)} \\
& \times(s-a)_{3}^{\lambda m+\rho+\nu} \psi_{2}\left[\begin{array}{c}
(\eta, \xi)(\gamma, \sigma)(1,1) \\
(\rho, m)(\lambda m+\rho+\nu+1, \mu)
\end{array} \mid-w(s-a)^{\mu}\right]
\end{aligned}
$$

Theorem 11. Consider $\quad \alpha, \beta, \mu, \nu, \eta, \rho, \gamma, \gamma, \lambda, \in \mathbb{C} \quad$ with $\quad \mathfrak{R}(\nu) \geq-1, \mathfrak{R}(\eta)>0, \mathfrak{R}(\rho)>0, \mathfrak{R}(\gamma)>0, \xi, m, \sigma \geq 0$, and $\mathfrak{R}(\gamma)>0, \mathfrak{R}(\alpha)>0, \mathfrak{R}(\beta)>-1, \quad q>0, \quad \mathfrak{R}(\mu)>0, \quad m, \xi>\mathfrak{R}(\mu)+\sigma$; then, the following relation holds:

$$
\begin{aligned}
{\left[D_{\nu, \eta, \rho, \gamma, w, 0^{+}}^{\mu, \xi, m, \sigma} \tau^{-1} J_{\beta}^{\alpha}(\tau) J_{\beta, q}^{\alpha, \gamma}\left(\tau^{-\lambda}\right)\right](s)=} & \frac{\Gamma(\rho)[\Gamma(\eta)]^{-1}}{\Gamma(\gamma) \Gamma(\gamma)} \sum_{m, n=0}^{\infty} \frac{\Gamma(\eta+\xi n) \Gamma(\gamma+\sigma n)}{\Gamma(\rho+m n)} \\
& \left.\times \frac{\left(-w s^{\mu}\right)^{n}(-s)^{m} s^{-\nu}}{\Gamma(\alpha m+\beta+1) m !^{2}} \psi_{2}\left[\begin{array}{l}
(\gamma, q)(m,-\lambda) \\
(\beta+1, \alpha)(\mu n+m-\nu+1,-\lambda)
\end{array}\right)-s^{-\lambda}\right]
\end{aligned}
$$

Proof. Consider inverse fractional integral operator (23) with the product of two Bessel-Maitland functions (1) and (2); then, the following results hold:

$$
\begin{aligned}
& {\left[D_{\nu, \eta, \rho, \gamma, w, 0^{+}}^{\mu, \xi, m, \sigma} \tau_{\beta}^{-1}(\tau) J_{\beta, q}^{\alpha, \gamma}\left(\tau^{-\lambda}\right)\right](s)} \\
& =\left(\frac{\mathrm{d}^{p}}{\mathrm{~d} s^{p}}\right) \int_{0}^{s}(s-\tau)^{p-\nu} \sum_{n=0}^{\infty} \frac{(\eta)_{\xi n}(\gamma)_{\sigma n}\left(-w(s-\tau)^{\mu}\right)^{n}}{(\rho)_{m n} \Gamma(\mu n+p-\nu+1)} \times \sum_{m=0}^{\infty} \frac{\tau^{-1}(-\tau)^{m}}{m ! \Gamma(\alpha m+\beta+1)} \sum_{w=0}^{\infty} \frac{(\gamma)_{q w}(-1)^{w}(\tau)^{-\lambda w}}{w ! \Gamma(\alpha w+\beta+1)} \mathrm{d} \tau \\
& =\left(\frac{\mathrm{d}^{p}}{\mathrm{~d} s^{p}}\right)_{\mu, p-\gamma}^{\gamma, \sigma} Q_{\rho, m ; n}^{\eta, \xi} \sum_{m, w=0}^{\infty} \frac{(-1)^{m+w} s^{p-\nu+\mu n}}{m ! \Gamma(\alpha m+\beta+1)} \\
& \quad \times \frac{(\gamma)_{q w}(w)^{n}}{w ! \Gamma(\alpha w+\beta+1)} \int_{0}^{s}\left(1-\frac{\tau}{s}\right)^{p-\nu+\mu n} \tau^{-1+m-\lambda w} \mathrm{~d} \tau .
\end{aligned}
$$

By substituting $u=(\tau / s)$ in equation (94), we have

$$
\begin{aligned}
& {\left[D_{\nu, \eta, \rho, \gamma, w, 0^{+}}^{\mu, \xi, m, \sigma} \tau^{-1} J_{\beta}^{\alpha}(\tau) J_{\beta, q}^{\alpha, \gamma}\left(\tau^{-\lambda}\right)\right](s)} \\
& \quad=\left(\frac{\mathrm{d}^{p}}{\mathrm{~d} s^{p}}\right)_{\mu, p-\nu}^{\gamma, \sigma} Q_{\rho, m ; n}^{\eta, \xi} \sum_{m, w=0}^{\infty} \frac{(w)^{n}(-1)^{m+w} s^{\mu n}}{m ! \Gamma(\alpha m+\beta+1)} \frac{(\gamma)_{q w} s^{m-\lambda w+p-\nu}}{w ! \Gamma(\alpha w+\beta+1)} \int_{0}^{1}(1-u)^{p-\nu+\mu n} u^{-1+m-\lambda w} \mathrm{~d} u .
\end{aligned}
$$


By using beta-gamma relations (11) and (12), we obtain

$$
\begin{aligned}
& {\left[D_{\nu, \eta, \rho, \gamma, w, 0^{+}}^{\mu, \xi, m, \sigma} \tau^{-1} J_{\beta}^{\alpha}(\tau) J_{\beta, q}^{\alpha, \gamma}\left(\tau^{-\lambda}\right)\right](s)} \\
& \quad={ }_{\mu, p-\nu}^{\gamma, \sigma} Q_{\rho, m ; n}^{\eta, \xi} \sum_{m, w=0}^{\infty} \frac{(w)^{n}(-1)^{m+w} s^{\mu n}}{m ! \Gamma(\alpha m+\beta+1)} \frac{(\gamma)_{q w} s^{m-\lambda w+p-\nu}}{w ! \Gamma(\alpha w+\beta+1)} \frac{\Gamma(p-\nu+\mu n+1) \Gamma(m-\lambda w)}{\Gamma(p-\nu+\mu n+m-\lambda w+1)} .
\end{aligned}
$$

Putting the value of ${ }_{\mu, p-v}^{\gamma, \sigma} Q_{\rho, m ; n}^{\eta, \xi}$ in equation (96), we have

$$
\begin{aligned}
{\left[D_{\nu, \eta, \rho, \gamma, w, 0^{+}}^{\mu, \xi, m, \sigma} \tau^{-1} J_{\beta}^{\alpha}(\tau) J_{\beta, q}^{\alpha, \gamma}\left(\tau^{-\lambda}\right)\right](s) } & \\
= & \sum_{m, n, w=0}^{\infty} \frac{(\eta)_{\xi n}(\gamma)_{\sigma n}(-w)^{n}\left(\mathrm{~d}^{p} / \mathrm{d} s^{p}\right)(s)^{p-\nu+\mu n+\lambda m+\rho}}{(\rho)_{m n} \Gamma(\mu n+\rho+\lambda m+p-\nu+1)} \frac{(w)^{n}(-1)^{m+w}}{m ! \Gamma(\alpha m+\beta+1)} \frac{(\gamma)_{q w} \Gamma(m-\lambda w)}{w ! \Gamma(\alpha w+\beta+1)} \\
= & \sum_{m, n, w=0}^{\infty} \frac{(\eta)_{\xi n}(\gamma)_{\sigma n}(-w)^{n}(s)^{-\nu+\mu n+\lambda m+\rho}}{(\rho)_{m n} \Gamma(\mu n+\rho+\lambda m-\nu+1)} \frac{(w)^{n}(-1)^{m+w}}{m ! \Gamma(\alpha m+\beta+1)} \frac{(\gamma)_{q w} \Gamma(m-\lambda w)}{w ! \Gamma(\alpha w+\beta+1)} \\
= & \frac{\Gamma(\rho)[\Gamma(\eta)]^{-1}}{\sum^{\infty} \frac{\Gamma(\eta+\xi n) \Gamma(\gamma+\sigma n)}{\Gamma(\rho+m n)} \frac{\left(-w s^{\mu}\right)^{n}(-s)^{m} s^{-v}}{\Gamma(\alpha m+\beta+1) m !}} \\
& \times{ }_{2} \psi_{2}\left[\begin{array}{l}
(\gamma, q)(m,-\lambda) \\
m, n=0
\end{array}\right]
\end{aligned}
$$

Corollary 2. If we replace $v=-v$ in Theorem 11 , we have the result in the sense of the left inverse operator:

$$
\begin{aligned}
{\left[\mathscr{Z}_{\nu, \eta, \rho, \gamma, w, 0^{+}}^{\mu, \xi, m, \sigma} \tau^{-1} J_{\beta}^{\alpha}(\tau) J_{\beta, q}^{\alpha, \gamma}\left(\tau^{-\lambda}\right)\right](s)=} & \frac{\Gamma(\rho)[\Gamma(\eta)]^{-1}}{\Gamma(\gamma) \Gamma(\gamma)} \sum_{m, n=0}^{\infty} \frac{\Gamma(\eta+\xi n) \Gamma(\gamma+\sigma n)}{\Gamma(\rho+m n)} \\
& \left.\times \frac{\left(-w s^{\mu}\right)^{n}(-s)^{m} s^{\nu}}{\Gamma(\alpha m+\beta+1) m !^{2}} \psi_{2}\left[\begin{array}{l}
(\gamma, q)(m,-\lambda) \\
(\beta+1, \alpha)(\mu n+m+\nu+1,-\lambda)
\end{array}\right]-s^{-\lambda}\right] .
\end{aligned}
$$

Theorem 12. Let $\alpha, \beta, \mu, \nu, \eta, \rho, \gamma \in \mathbb{C}, \quad \mathfrak{R}(\mu)>0$, $\mathfrak{R}(\nu) \geq-1, \mathfrak{R}(\eta)>0, \mathfrak{R}(\rho)>0, \mathfrak{R}(\gamma)>0, \xi, m, \sigma \geq 0$, and $m, \xi>\Re(\mu)+\sigma$; then, there exists the following relation:

$$
\begin{aligned}
& {\left[D_{\nu, \eta, \rho, \gamma, w, 0^{+}}^{\mu, \xi, m, \sigma}{ }_{2}^{(\alpha / \beta)-1} R_{1}\left(\frac{\alpha}{\beta}+\mu,-\eta: \frac{\alpha}{\beta}: \tau\right)\right](s)} \\
& \quad=\sum_{m, n=0}^{\infty} \frac{((\alpha / \beta)+\mu)_{m}(-\eta)_{m}}{m !(-\nu+\mu n+(\alpha / \beta)+1)_{m}} \times \frac{\Gamma(\eta+\xi n) \Gamma(\gamma+\sigma n) s^{-\nu+m+(\alpha / \beta)}}{\Gamma(-\nu+\mu n+(\alpha / \beta)+1) \Gamma(\rho+m n)} \frac{\left(-w s^{\mu}\right)^{n} \Gamma(\rho)}{\Gamma(\eta) \Gamma(\gamma)} .
\end{aligned}
$$


Proof. Consider fractional integral operator (22) with Gauss hypergeometric function (8); then, the following results hold:

$$
\begin{aligned}
& {\left[D_{\nu, \eta, \rho, \gamma, w, 0^{+}}^{\mu, \xi, m, \sigma} \tau^{\alpha / \beta} R_{1}\left(\frac{\alpha}{\beta}+\mu,-\eta ; \frac{\alpha}{\beta} ; \tau\right)\right](s)} \\
& \quad=\left(\frac{\mathrm{d}^{p}}{\mathrm{~d} s p}\right) \int_{0}^{s}(s-\tau)^{p-\nu} \sum_{n=0}^{\infty} \frac{(\eta)_{\xi n}(\gamma)_{\sigma n}\left(-w(s-\tau)^{\mu}\right)^{n}}{(\rho)_{m n} \Gamma(\mu n+p-\nu+1)} \tau^{(\alpha / \beta)-1} \sum_{m=0}^{\infty} \frac{((\alpha / \beta)+\mu)_{m}(-\eta)_{m}}{(\alpha / \beta)_{m} m !}(\tau)^{m} \mathrm{~d} \tau \\
& \quad=\left(\frac{\mathrm{d}^{p}}{\mathrm{~d} s^{p}}\right)_{\mu, p-\gamma}^{\gamma, \sigma} Q_{\rho, m ; n}^{\eta, \xi}(w)^{n} \sum_{m=0}^{\infty} \frac{((\alpha / \beta)+\mu)_{m}(-\eta)_{m} s^{p-\nu+\mu n}}{(\alpha / \beta)_{m} m !} \int_{0}^{s}\left(\frac{1-\tau}{s}\right)^{p-\gamma+\mu n} \tau^{m+(\alpha / \beta)-1} \mathrm{~d} \tau .
\end{aligned}
$$

Putting $u=(\tau / s)$ in equation (100), we get

$$
\begin{aligned}
& {\left[D_{\nu, \eta, \rho, \gamma, w, 0^{+}}^{\mu, \xi, m, \sigma} \frac{\tau^{\alpha / \beta}}{\tau}{ }_{2} R_{1}\left(\frac{\alpha}{\beta}+\mu,-\eta ; \frac{\alpha}{\beta} ; \tau\right)\right](s)} \\
& \quad=\left(\frac{\mathrm{d}^{p}}{\mathrm{~d} s^{p}}\right)_{\mu, p-\nu}^{\gamma, \sigma} Q_{\rho, m ; n}^{\eta, \xi}(w)^{n} \sum_{m=0}^{\infty} \frac{((\alpha / \beta)+\mu)_{m}(-\eta)_{m} s^{p-\gamma+\mu n}}{(\alpha / \beta)_{m} m ! s^{-m-(\alpha / \beta)}} \int_{0}^{1}(1-u)^{p-\nu+\mu n} u^{m+(\alpha / \beta)-1} \mathrm{~d} u .
\end{aligned}
$$

Using equations (11) and (12), we have

$$
\begin{aligned}
& {\left[D_{\nu, \eta, \rho, \gamma, w, 0^{+}}^{\mu, \xi} \frac{\tau^{\alpha / \beta}}{\tau}{ }_{2} R_{1}\left(\frac{\alpha}{\beta}+\mu,-\eta ; \frac{\alpha}{\beta} ; \tau\right)\right](s)} \\
& \quad=\left(\frac{\mathrm{d}^{p}}{\mathrm{~d} s^{p}}\right)_{\mu, p-\nu}^{\gamma, \sigma} Q_{\rho, m ; n}^{\eta, \xi}(w)^{n} \sum_{m=0}^{\infty} \frac{((\alpha / \beta)+\mu)_{m}(-\eta)_{m} s^{p-\nu+\mu n}}{(\alpha / \beta)_{m} m ! s^{-m-(\alpha / \beta)}} \frac{\Gamma(p-v+\mu n+1) \Gamma(m+(\alpha / \beta))}{\Gamma(p-v+\mu n+m+(\alpha / \beta)+1)} .
\end{aligned}
$$

By substituting $\underset{\mu, p-v}{\gamma, \sigma} Q_{\rho, m ; n}^{\eta, \xi}$ in equation (102), we get the required result:

$$
\begin{aligned}
& {\left[D_{\nu, \eta, \rho, \gamma, w, 0^{+}}^{\mu, \xi, \tau_{2}(\alpha / \beta)-1} R_{1}\left(\frac{\alpha}{\beta}+\mu,-\eta ; \frac{\alpha}{\beta} ; \tau\right)\right](s)} \\
& \quad=\sum_{n, m=0}^{\infty} \frac{(\eta)_{\xi n}(\gamma)_{\sigma n}(-w)^{n} \Gamma(m+(\alpha / \beta))(-\eta)_{m}}{(\rho)_{m n} \Gamma(p-\nu+\mu n+m+(\alpha / \beta)+1)} \frac{((\alpha / \beta)+\mu)_{m}}{(\alpha / \beta)_{m} m !} \frac{\mathrm{d}^{p}}{\mathrm{~d} s^{p}} s^{p-\nu+\mu n+m+(\alpha / \beta)} \\
& \quad=\sum_{m, n=0}^{\infty} \frac{((\alpha / \beta)+\mu)_{m}(-\eta)_{m}}{m !(-\nu+\mu n+(\alpha / \beta)+1)_{m}} \frac{\left(-w s^{\mu}\right)^{n} \Gamma(\rho)}{\Gamma(\eta) \Gamma(\gamma)} \frac{\Gamma(\gamma+\sigma n)}{\Gamma(\rho+m n)} \frac{\Gamma(\eta+\xi n) s^{-\nu+m+(\alpha / \beta)}}{\Gamma(-\nu+\mu n+(\alpha / \beta)+1)}
\end{aligned}
$$

Corollary 3. By putting $v=-v$ in Theorem 12, we get the following form:

$$
\begin{aligned}
{\left[\mathscr{Z}_{\nu, \eta, \rho, \gamma, w, 0^{+}}^{\mu, \xi, m, \sigma} \tau_{2}^{(\alpha / \beta)-1} R_{1}\left(\frac{\alpha}{\beta}+\mu,-\eta ; \frac{\alpha}{\beta} ; \tau\right)\right](s)=} & \sum_{m, n=0}^{\infty} \frac{((\alpha / \beta)+\mu)_{m}(-\eta)_{m}}{m !(\nu+\mu n+(\alpha / \beta)+1)_{m}} \\
& \times \frac{\Gamma(\eta+\xi n) \Gamma(\gamma+\sigma n) s^{\nu+m+(\alpha / \beta)}}{\Gamma(\nu+\mu n+(\alpha / \beta)+1) \Gamma(\rho+m n)} \frac{\left(-w s^{\mu}\right)^{n} \Gamma(\rho)}{\Gamma(\eta) \Gamma(\gamma)} .
\end{aligned}
$$




\section{Conclusion}

In this paper, we discussed some relations of generalized Bessel-Maitland functions and the Mittag-Leffler functions and also developed Saigo and Riemann-Liouville fractional integral operators with the generalized Bessel-Maitland function, and results can be seen in the form of Fox-Wright functions. Also, we established a new operator $\mathscr{Z}_{\nu, \eta, p, \gamma, w, a^{+}}^{\mu, \xi} \phi$ and also discussed its convergence and boundedness. Moreover, we discussed the Riemann-Liouville fractional operator and the integral transform (Laplace) of the new operator and also developed some important applications of the left inverse operator.

\section{Data Availability}

No data were used to support this study since they are more of simulation.

\section{Conflicts of Interest}

The authors declare that they have no conflicts of interest.

\section{References}

[1] P. Agarwal, "Pathway fractional integral formulas involving Bessel function of the first kind," Advanced Studies in Contemporary Mathematics, vol. 25, no. 1, pp. 221-231, 2015.

[2] P. Agarwal, S. Jain, M. Chand, S. K. Dwivedi, and S. Kumar, "Bessel functions associated with Saigo-Maeda fractional derivative operators," Journal of Fractional Calculus, vol. 5, no. 2, pp. 102-112, 2014.

[3] Y. A. Brychkov, Handbook of Special Functions: Derivatives, Integrals, Series and Other Formulas, Chapman and Hall/ CRC, London, UK, 2008.

[4] W. A. Khan and K. S. Nisar, "Unified integral operator involving generalized Bessel-Maitland function," 2017, https:// arxiv.org/abs/1704.09000.

[5] M. S. Abouzaid, A. H. Abusufian, and K. S. Nisar, "Some unified integrals associated with generalized Bessel-Maitland function," 2016, https://arxiv.org/abs/1605.09200.

[6] G. N. Watson, A Treatise on the Theory of Bessel Functions, Cambridge University Press, Cambridge, UK, 1995.

[7] M. Singh, M. A. Khan, M. Ahmad Khan, and A. Hakim Khan, "On some properties of a generalization of Bessel-Maitland function," International Journal of Mathematics Trends and Technology, vol. 14, no. 1, pp. 46-54, 2014.

[8] D. L. Suthar, A. M. Khan, A. Alaria, S. D. Purohit, and J. Singh, "Extended Bessel-Maitland function and its properties pertaining to integral transforms and fractional calculus," AIMS Mathematics, vol. 5, no. 2, p. 1400, 2020.

[9] M. Kamarujjama, N. U. Khan, and O. Khan, "Estimation of certain integrals with extended multi-index Bessel function," Malaya Journal of Matematik, vol. 7, no. 2, pp. 206-212, 2019.

[10] S. D. Purohit, P. Agarwal, and S. Mathur, "Certain new integral formulas involving the generalized Bessel functions," Bulletin of the Korean Mathematical Society, vol. 51, no. 4, pp. 995-1003, 2014.

[11] Y. L. Luke, Integrals of Bessel Functions, Courier Corporation, Chelmsford, MA, USA, 2014.

[12] J. Choi and P. Agarwal, "Certain unified integrals associated with Bessel functions," Boundary Value Problems, vol. 2013, no. 1, p. 95, 2013.
[13] M. Ali and W. A. Khan, "Some integral involving extended Bessel-Maitland function with Jacobi polynomial," GANITA, vol. 69, no. 2, 2019.

[14] D. L. Suthar and H. Amsalu, "Certain integrals associated with the generalized Bessel-Maitland function," Applications and Applied Mathematics, vol. 12, no. 2, 2017.

[15] P. Agarwal, Q. Al-Mdallal, Y. J. Cho, and S. Jain, "Fractional differential equations for the generalized Mittag-Leffler function," Advances in Difference Equations, vol. 2018, no. 1, p. 58, 2018.

[16] Q. Al-Mdallal, M. Al-Refai, M. Syam, and M. D. K. Al-Srihin, "Theoretical and computational perspectives on the eigenvalues of fourth-order fractional Sturm-Liouville problem," International Journal of Computer Mathematics, vol. 95, no. 8, pp. 1548-1564, 2018.

[17] T. Abdeljawad and Q. M. Al-Mdallal, "Discrete Mittag-Leffler kernel type fractional difference initial value problems and Gronwall's inequality," Journal of Computational and Applied Mathematics, vol. 339, pp. 218-230, 2018.

[18] T. Abdeljawad, Q. M. Al-Mdallal, and M. A. Hajji, “Arbitrary order fractional difference operators with discrete exponential kernels and applications," Discrete Dynamics in Nature and Society, vol. 2017, Article ID 4149320, 8 pages, 2017.

[19] O. I. Merichev, Handbook of Integral Transforms and Higher Transcendental Functions. Theory and Algorithm Tables, John Wiley and Sons, New York, NY, USA, 1983.

[20] M. Ghayasuddin and W. A. Khan, "A new extension of BesselMaitland function and its properties," Matematicki Vesnik, vol. 70, no. 4, pp. 292-302, 2018.

[21] M. Saigo, "A remark on integral operators involving the Gauss hypergeometric functions," Mathematical Reports, Kyushu University, vol. 11, pp. 135-143, 1978.

[22] S. G. Samko, A. A. Kilbas, and O. I. Marichev, Fractional Integrals and Derivatives. Theory and Application, Gordon and Breach Science Publishers, Yverdon, Switzerland, 1993.

[23] A. Petojevic, "A note about the Pochhammer symbol," Mathematica Moravica, vol. 12, no. 1, pp. 37-42, 2008.

[24] E. D. Rainville, The Laplace Transform: An Introduction, Macmillan Publishers, New York, NY, USA, 1963.

[25] S. Ahmed, "On the generalized fractional integrals of the generalized Mittag-Leffler function," SpringerPlus, vol. 3, no. 1, p. 198, 2014.

[26] F. Polito and Z. Tomovski, "Some properties of Prabhakartype fractional calculus operators," 2016, https://arxiv.org/ abs/1508.03224.

[27] T. O. Salim and A. W. Faraj, "A generalization of MittagLeffler function and integral operator associated with fractional calculus," Journal of Fractional Calculus and Applications, vol. 3, no. 5, pp. 1-13, 2012.

[28] A. K. Shukla and J. C. Prajapati, "On a generalization of MittagLeffler function and its properties," Journal of Mathematical Analysis and Applications, vol. 336, no. 2, pp. 797-811, 2007.

[29] H. M. Srivastava and Ž. Tomovski, "Fractional calculus with an integral operator containing a generalized Mittag-Leffler function in the kernel," Applied Mathematics and Computation, vol. 211, no. 1, pp. 198-210, 2009.

[30] T. R. Prabhakar, "A singular integral equation with a generalized Mittag Leffler function in the kernel," Yokohama Mathematical Journal, vol. 19, pp. 7-15, 1971.

[31] A. Wiman, "Über den fundamentalsatz in der teorie der funktionen $E_{a}(x)$, , Acta Mathematica, vol. 29, no. 1, pp. 191-201, 1905.

[32] G. M. Mittag-Leffler, "Sur la nouvelle fonction $E_{a}(x)$," Comptes Rendus de l'Académie des Sciences, vol. 137, no. 2, pp. 554-558, 1903. 